\title{
Microglia convert aggregated amyloid- $\beta$ into neurotoxic forms through the shedding of microvesicles
}

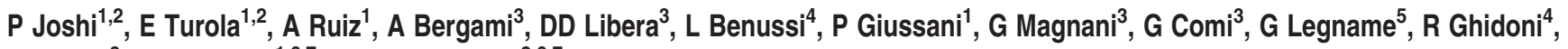 \\ R Furlan ${ }^{3}$, M Matteoli*, $^{\star 1,6,7}$ and C Verderio*,2,6,7
}

\begin{abstract}
Alzheimer's disease (AD) is characterized by extracellular amyloid- $\beta(A \beta)$ deposition, which activates microglia, induces neuroinflammation and drives neurodegeneration. Recent evidence indicates that soluble pre-fibrillar $A \beta$ species, rather than insoluble fibrils, are the most toxic forms of $A \beta$. Preventing soluble $A \beta$ formation represents, therefore, a major goal in AD. We investigated whether microvesicles (MVs) released extracellularly by reactive microglia may contribute to AD degeneration. We found that production of myeloid MVs, likely of microglial origin, is strikingly high in AD patients and in subjects with mild cognitive impairment and that AD MVs are toxic for cultured neurons. The mechanism responsible for MV neurotoxicity was defined in vitro using MVs produced by primary microglia. We demonstrated that neurotoxicity of MVs results from (i) the capability of MV lipids to promote formation of soluble $A \beta$ species from extracellular insoluble aggregates and (ii) from the presence of neurotoxic $A \beta$ forms trafficked to MVs after $A \beta$ internalization into microglia. MV neurotoxicity was neutralized by the $A \beta$-interacting protein PrP and anti-A $\beta$ antibodies, which prevented binding to neurons of neurotoxic soluble $A \beta$ species. This study identifies microglia-derived MVs as a novel mechanism by which microglia participate in AD degeneration, and suggest new therapeutic strategies for the treatment of the disease.
\end{abstract}

Cell Death and Differentiation (2014) 21, 582-593; doi:10.1038/cdd.2013.180; published online 13 December 2013

Alzheimer's disease (AD) is the major cause of dementia in humans. Neuronal loss and cognitive decline occurring in AD patients are traditionally linked to the accumulation in the brain of extracellular plaques consisting of short amyloid- $\beta(\mathrm{A} \beta)$ peptides of 39-42 amino acids, generated by amyloidogenic cleavage of the amyloid precursor protein. ${ }^{1}$ Among $\mathrm{A} \beta$ peptides, $\mathrm{A} \beta$ 1-42 and pyroglutamate-modified $\mathrm{A} \beta$ very rapidly aggregate and initiate the complex multistep process that leads to mature fibrils and plaque., ${ }^{2,3}$

Although association of amyloid plaques with $A D$ has long been assumed, $\mathrm{A} \beta$ load does not correlate with neuronal loss $^{4,5}$ and high plaque burden does not necessarily lead to dementia in humans. ${ }^{6,7}$ Accordingly, recent evidence clearly showed that the amyloid load reaches a plateau early after the onset of clinical symptoms in $A D$ patients ${ }^{8}$ and does not substantially increase in size during clinical progression. ${ }^{9}$ These observations agree with the current view that small, soluble pre-fibrillar $\mathrm{A} \beta$ species, rather than plaques formed by insoluble $\mathrm{A} \beta$ fibrils, are the most toxic forms of $\mathrm{A} \beta .^{10}$ These cause synaptic dysfunction and spine loss, and correlate most closely with the severity of human AD. ${ }^{5,8,11}$ Recent biochemical studies indicated that natural sphingolipids and gangliosides, whose metabolism has been shown to be altered in AD patients, ${ }^{12}$ destabilize and rapidly resolubilize long $A \beta$ fibrils to neurotoxic species. ${ }^{13}$ These studies also showed that phospholipids stabilize toxic oligomers from monomeric peptides. $^{14}$

The toxicity of small soluble $\mathrm{A} \beta$ species has been proposed to depend on the interaction with specific neuronal proteins, such as the NMDA receptor ${ }^{15}$ or the prion protein $\left(\operatorname{PrP}^{\mathrm{C}}\right){ }^{16}$ which modulates NMDA receptors through Fyn kinase. ${ }^{17}$ Alternatively, soluble $\mathrm{A} \beta$ oligomers may damage neurons by binding to multiple membrane components, including lipids, thereby changing membrane permeability and causing calcium ion leakage into the cell. ${ }^{5,18}$

Neuroinflammation arguably has a role in promoting neurotoxicity of $A \beta$ plaques. This is suggested by several lines of evidence: (i) subjects with high plaque burden without dementia show virtually no evidence of neuroinflammation; ${ }^{6}$ (ii) recent PET studies ${ }^{19,20}$ showed an inverse correlation

\footnotetext{
${ }^{1}$ Department of Biotechnology and Translational Medicine, University of Milano, via Vanvitelli 32, Milano 20129, Italy; ${ }^{2}$ Department of Medicine, CNR Institute of Neuroscience, via Vanvitelli 32, Milano 20129, Italy; ${ }^{3}$ INSPE, Division of Neuroscience, San Raffaele Scientific Institute, via Olgettina 60, Milano 20132, Italy; ${ }^{4}$ Proteomics Unit, IRCCS Istituto centro San Giovanni di Dio Fatebenefratelli, via Pilastroni, Brescia 4 25125, Italy; ${ }^{5}$ SISSA, Department of Neuroscience, Via Bonomea 265, Trieste I-34136, Italy and ${ }^{6}$ IRCCS Humanitas, via Manzoni 56, Rozzano 20089, Italy

${ }^{*}$ Corresponding authors: M Matteoli, Department of Biotechnology and Translational Medicine, Via Vanvitelli 32, Milano 20129, Italy. Tel: +390250317095 ; Fax: +390250317132; E-mail: michela.matteoli@unimi.it

or C Verderio, CNR Institute of Neuroscience, Via Vanvitelli 32, Milano 20129, Italy.Tel: +390250317098; Fax: +390250317132; E-mail: c.verderio@ in.cnr.it ${ }^{7} J o i n t$ senior authors.

Keywords: microglia; extracellular microvesicles; Abeta 1-42; prion protein; bioactive lipids; Alzheimer's disease

Abbreviations: EMVs, extracellular membrane microvesicles; MVs, microvesicles; AA-MVs, acutely added MVs; A $\beta$, amyloid- $\beta$; AD, Alzheimer's disease; MCl, mild cognitive impairment; $\mathrm{PrP}^{\mathrm{C}}$, prion protein; CSF, cerebrospinal fluid; PI, propidium iodide; $\mathrm{KRH}$, Krebs-Ringer solution; ThT, thioflavin-T

Received 18.6.13; revised 15.10.13; accepted 30.10.13; Edited by L Greene; published online 13.12 .13
} 
between the cognitive status and activation of microglia, the immune cells of the nervous system, in $A D$ patients; (iii) activation of microglia increases linearly throughout the disease course and correlates with AD neurodegeneration. ${ }^{8}$ Moreover, recent studies demonstrating that variants of TREM2 and CD33, two receptors expressed in microglial cells, increase the risk for late-onset $A D^{21,22}$ have refocused the spotlight on microglia as a major contributing factor in AD. Although multiple preclinical evidence indicates that microglia activation promotes neuronal dysfunction and neuron elimination $^{23,24}$ and accelerates AD progression, ${ }^{19,25,26}$ the molecular mechanisms by which microglia exert neurotoxicity remain largely unknown.

We have recently described a novel mechanism of cell-tocell communication in the brain, by which reactive microglia propagate an inflammatory signal through the release of extracellular membrane microvesicles (EMVs), which bud from the cell surface, called shed microvesicles (MVs) or ectosomes. MVs are shed by microglia upon ATP activation ${ }^{27}$ and originate from lipid rafts, ${ }^{28}$ where the ATP receptor $\mathrm{P}_{2} \mathrm{X}_{7}$ is localized. ${ }^{29}$ Shed MVs selectively accumulate various cellular components, including soluble and integral proteins, lipids and nucleic acids and their composition reflects the activation state of donor microglia. Notably, microglia-derived MVs in the cerebrospinal fluid (CSF) have been recently identified as a novel biomarker of brain inflammation in humans. ${ }^{30,31}$

The observation that typical proteins of EMVs, like flotilin, accumulate in the plaques of $\mathrm{AD}$ brain, ${ }^{32}$ together with evidence that activated microglia constantly surround amyloid deposits, ${ }^{33}$ prompted us to investigate whether EMVs may be involved in the spatiotemporal propagation of $\mathrm{A} \beta$ pathology through the brain. Here we show that production of MVs is extremely high in patients with $A D$ and that microglial MVs, either shed in vitro or isolated from the CSF of AD patients, promote generation of soluble neurotoxic $A \beta$ species, thereby acting as potent drivers of neuronal damage.

\section{Results}

The combination of $A \beta$ 1-42 and microglia-derived MVs is neurotoxic in vitro. The evidence that natural lipids may shift the equilibrium between insoluble and soluble $\mathrm{A} \beta$ toward highly toxic soluble species ${ }^{13,34}$ prompted us to test whether MVs shed from microglial cells may promote $\mathrm{A} \beta$ neurotoxicity. A $\beta$ 1-42 (4 $\mu \mathrm{M})$ dissolved in dimethyl sulfoxide (DMSO) was incubated overnight with MVs derived from rat primary microglia $(1 \mu \mathrm{g} / 100 \mu \mathrm{l})$ at $37^{\circ} \mathrm{C}$ in neuronal medium and subsequently exposed to cultured hippocampal neurons for $1 \mathrm{~h}$. Overnight pre-incubation of $\mathrm{A} \beta 1-42$ with $\mathrm{MVs}$ yielded a neurotoxic mixture that significantly increased the percentage of dead neurons, as assessed $24 \mathrm{~h}$ later by propidium iodide (PI) and calcein staining (Figures 1a and b; number of experiments $=4)$. Notably, neither MVs alone nor MVs incubated overnight with scrambled $\mathrm{A} \beta$ 1-42 significantly affected neuronal survival (Figure 1b). A $\beta$ 1-42 alone, dissolved in DMSO and incubated overnight at $37^{\circ} \mathrm{C}$ in neuronal medium in the absence of MVs, from now on called aggregated $\mathrm{A} \beta$ 1-42 barely affected neuronal viability, even when supplemented with MVs just before neuron challenge (acutely added MVs, AA-MVs- Figure 1b). Collectively, these findings indicate that overnight pre-incubation of aggregated $\mathrm{A} \beta$ 1-42 with $\mathrm{MVs}$ is required for the development of neurotoxicity. A $\beta$ 1-42 pre-incubated with MVs induced cell death very rapidly. One hour after exposure to $\mathrm{A} \beta 1-42$ preincubated with MVs, about $15-30 \%$ of neurons loaded with the calcium dye Fura-2 displayed an abnormally high level of cytosolic calcium (Figures $1 \mathrm{~b}$ and $\mathrm{c} ; n=10$ ) and were positive for the early apoptotic marker annexin-V (Figure 1e; $n=6$ ). MVs alone, pre-incubated overnight in neuronal medium, also induced a slight increase in intracellular calcium concentrations (Figure 1d). Immunofluorescence analysis with the neuronal marker $\beta$-3 tubulin and the pre- and post-synaptic markers V-Glut-1 and PSD-95 revealed that processes of neurons treated with combined A $\beta$ 1-42 and MVs were fragmented and showed reduced synaptic density (Figures if and $\mathrm{g}$ ). Dendrite damage was associated to a marked decrease of MAP-2 immunoreactivity (Figure $3 \mathrm{f}$ ). The toxic effect of $\mathrm{A} \beta 1-42$ in combination with MVs was largely prevented when neurons were exposed to the mixture in the presence of the glutamate receptors antagonists APV $(100 \mu \mathrm{M})$ and CNQX $(20 \mu \mathrm{M})$, as evaluated by quantification of cytoplasmic calcium $(n=4)$, annexin-V $(n=2)$ or $\mathrm{Pl} /$ calcein $(n=2)$ staining (Figures $1 \mathrm{~h}-\mathrm{j})$. This suggests excitotoxic damage as the cause of neuronal death.

Shed MVs promote formation of soluble forms of A $\beta$ 1-42. A $\beta$ 1-42 pre-incubated overnight with MVs was partitioned into two phases by centrifugation for $30 \mathrm{~min}$ at $10000 \times g$. The neurotoxicity of the two fractions was analyzed by monitoring cytosolic calcium in cultured neurons. Whereas the supernatant retained a high degree of toxicity, the pellet was largely inert (Figure 2a left; $n=3$ ). Similar results were obtained by quantification of dead neurons by $\mathrm{Pl} /$ calcein assay (Figure $2 \mathrm{a}$ right; $n=3$ ). This suggested that soluble molecules, not associated to MVs, either generated from $\mathrm{A} \beta$ 1-42 synthetic peptides or deriving from MVs were mainly responsible for toxicity.

The inflammatory mediators IL- $1 \beta$ and TNF $\alpha$ are among the molecules contained in microglial MVs that, through potentiation of NMDA channel activity, may induce excitoxicity. ${ }^{27,35}$ As IL- $1 \beta$ and TNF $\alpha$ expression is upregulated in M1 proinflammatory microglia, and downregulated in $\mathrm{M} 2$ anti-inflammatory cells, we analyzed neuron viability after exposure to $\mathrm{A} \beta$ 1-42 incubated with MVs produced by either LPS-primed M1 microglia or M2 cells, polarized with IL-4. Similar alterations of cytoplasmic calcium were observed in neurons exposed to $\mathrm{A} \beta$ 1-42 in combination with MVs derived from M1 or M2 microglia (Figure $2 \mathrm{~b} ; n=3$ ) or exposed to the neurotoxic mixture in the presence of IL-1 $\beta$ - and TNF $\alpha$-neutralizing antibodies (Figure 2c; $n=4)$. These data rule out the possibility that excitotoxicity of $A \beta$ 1-42 in combination with MVs depends on cytokine leakage from MVs.

We then investigated whether neurotoxicity of $A \beta 1-42 /$ MVs mixture could be due to the presence of neurotoxic soluble $\mathrm{A} \beta$ forms. Negative staining electron microscopic analysis revealed the presence of both globular structures of diameter between 4 and 8 and $5-8 \mathrm{~nm}$ wide $\mathrm{A} \beta$ fibrils in samples of aggregated $\mathrm{A} \beta$ 1-42 incubated overnight with MVs. After fractionation into two phases by centrifugation, 
a
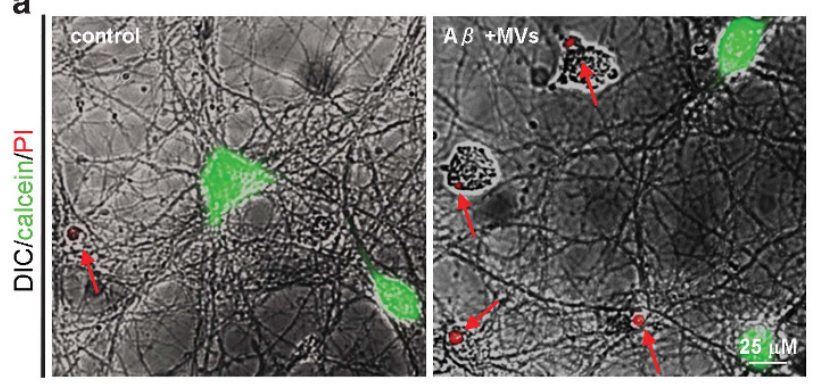

b

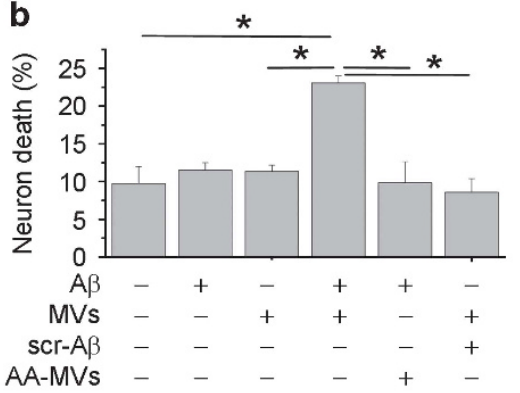

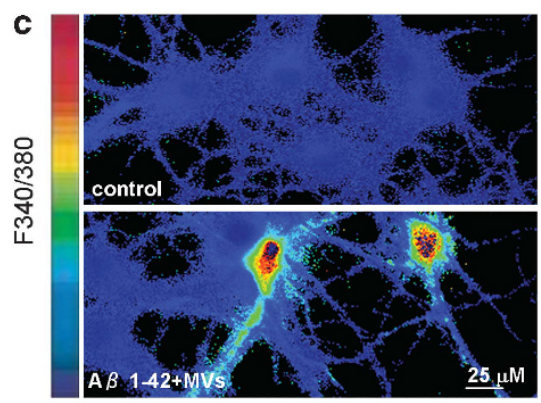

f

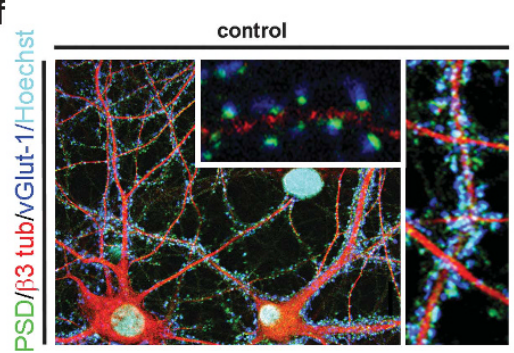

d

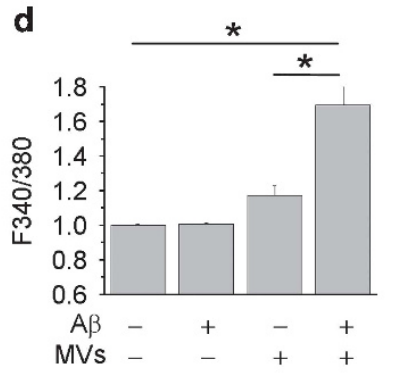

$A \beta+M V s$

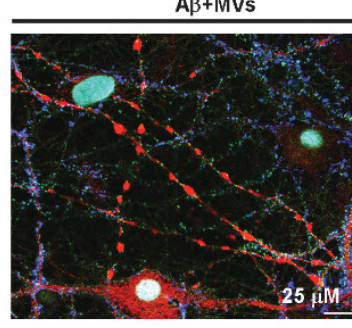

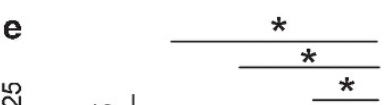

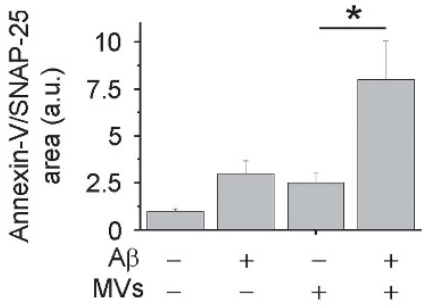

9

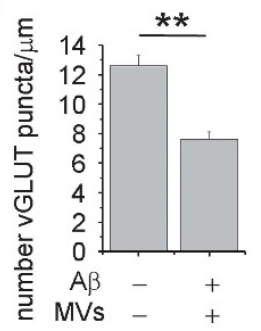

h

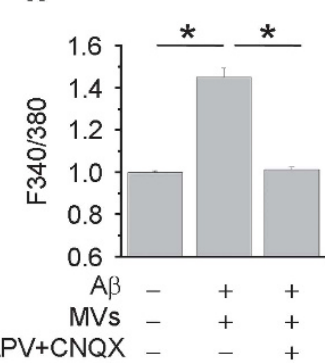

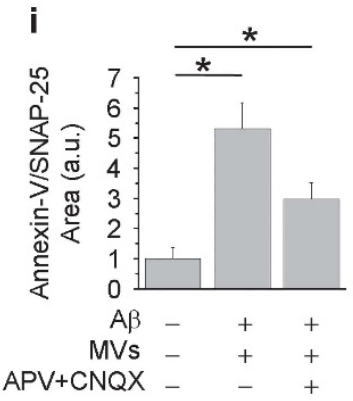

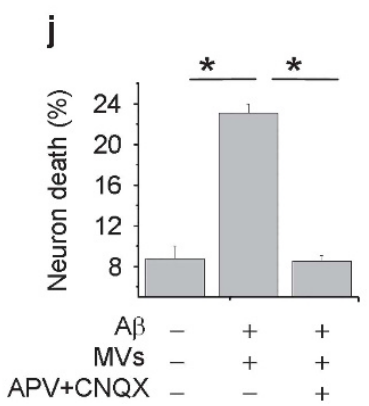

Figure 1 Microglia-derived MVs promote $\mathrm{A} \beta$ neurotoxicity. Fourteen DIV hippocampal neurons were exposed for $1 \mathrm{~h}$ to $\mathrm{A} \beta 1-42$ or scrambled $\mathrm{A} \beta 1-42(4 \mu \mathrm{M})$ preincubated with MVs $(1 \mu \mathrm{g} / 100 \mu \mathrm{l})$ overnight in neuronal medium. (a) Overlays of DIC and fluorescence microscopic images of neurons stained for calcein and propidium iodide $(\mathrm{PI})$, after $24 \mathrm{~h}$ exposure to $\mathrm{A} \beta 1-42 / \mathrm{MV}$ s mixture or under control conditions. (b) Percentage of calcein $-/ \mathrm{PI}+$ neurons (dead cells) in cultures exposed to $\mathrm{A} \beta$ 1-42, scrambled $A \beta 1-42$, MVs or $A \beta 1-42 /$ scrambled $A \beta 1-42$ incubated overnight with MVs. AA-MVs refer to freshly isolated MVs added to $A \beta 1-42$, just before neuron challenge (the Kruskal-Wallis ANOVA, $P<0.001$; Dunn's test for comparison among groups, ${ }^{*} P<0.05$ ). (c) Basal $\left[\mathrm{Ca}^{2+}\right]_{i}$ was measured in single neurons loaded with the ratiometric calcium dye Fura-2 and expressed as F340/380 fluorescence. Representative pseudocolor images of 9DIV control neurons and neurons treated with A $\beta 1-42 / M V s$ mixture for $1 \mathrm{~h}$. The color scale is shown on the left. (d) Quantification of basal $\left[\mathrm{Ca}^{2+}\right]_{i}$ in neurons exposed to A $\beta 1-42, \mathrm{MVs}$ or A $\beta 1-42$ in combination with MVs. At least 100 neurons/ condition were examined. Values are normalized to control (the Kruskal-Wallis ANOVA, $P=0.002$; Dunn's test for comparison among groups, ${ }^{*} P<0.05$ ). (e) Quantification of early apoptotic damage, revealed by Annexin-V binding, normalized to SNAP-25 immunoreactive area, in neurons treated as in $\mathrm{d}$. (the Kruskal-Wallis ANOVA, $P=0.001$; Dunn's test for comparison among groups, ${ }^{*} P<0.05$ ). (f) Confocal microscopic images of 14DIV neurons untreated or pretreated with A $\beta 1-42$ in combination with MVs and stained for $\beta$-3 tubulin, the vesicular glutamate transporter vGlut-1 and the postsynaptic marker PSD-95. Nuclei are stained with Hoechst. Note, fragmentation of neuronal processes and loss of excitatory synapses in neurons exposed to $A \beta 1-42 / M V s$ mixture. Density of excitatory synaptic puncta is quantified in $\mathbf{g}$ (data follow normal distribution, Student's $t$-test, $\left.{ }^{*} P<0.001\right)$. (h-j) Control cultures and cells treated with $\mathrm{A} \beta 1-42 / \mathrm{MV}$ s mixture analyzed for basal $\left[\mathrm{Ca}^{2+}\right]_{i}(\mathrm{~h}$, the Kruskal-Wallis ANOVA, $P=0.001$; Dunn's test for comparison among groups, ${ }^{*} P<0.05$ ), early apoptotic damage (i, the Kruskal-Wallis ANOVA, $P=0.001$; Dunn's test for comparison among groups, $\left.{ }^{\star} P<0.05\right)$ and calcein/PI staining (j, the Kruskal-Wallis ANOVA $P<0.001$; Dunn's test for comparison among groups, $\left.{ }^{*} P<0.05\right)$, either in the presence or in the absence of the glutamate receptor antagonists APV and CNQX 
a

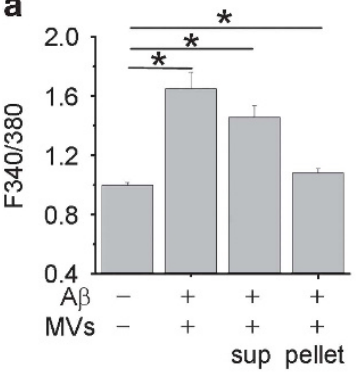

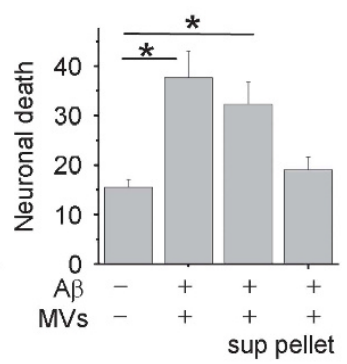

b

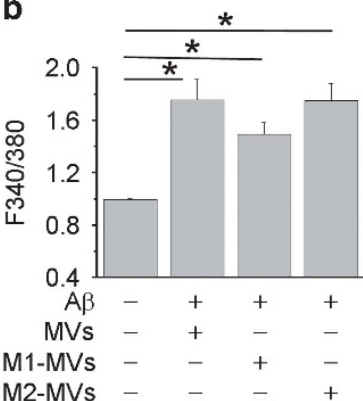

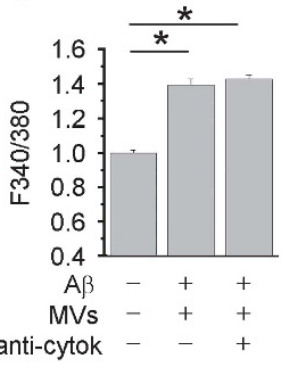

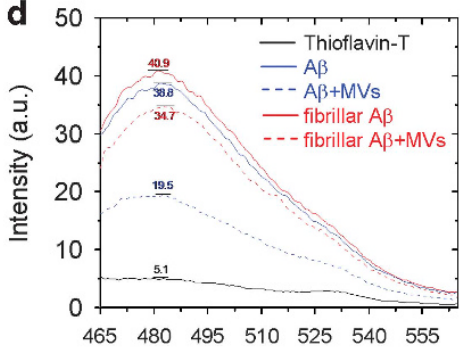

$\lambda(\mathrm{nm})$
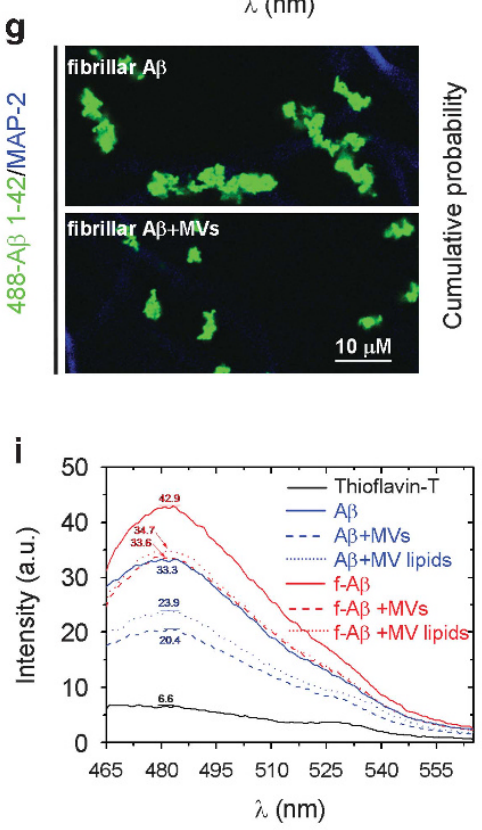

e 1

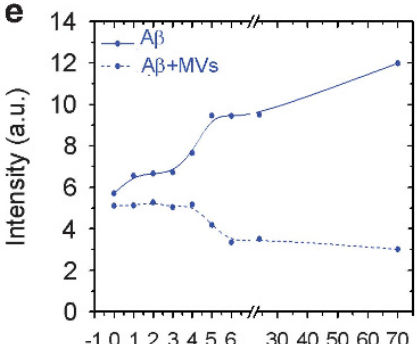

f

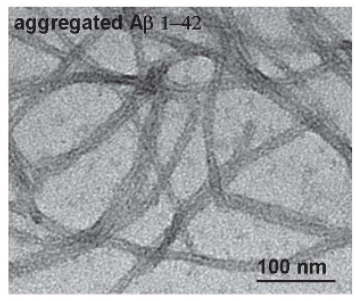

Time (hours)

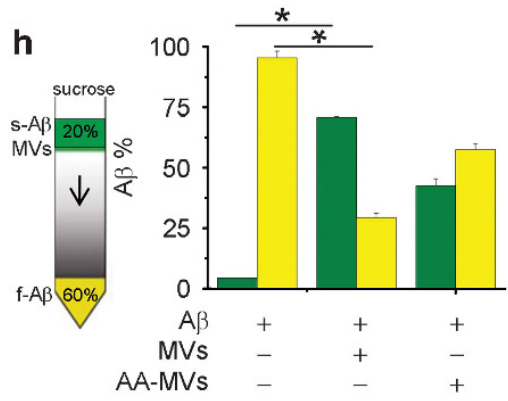

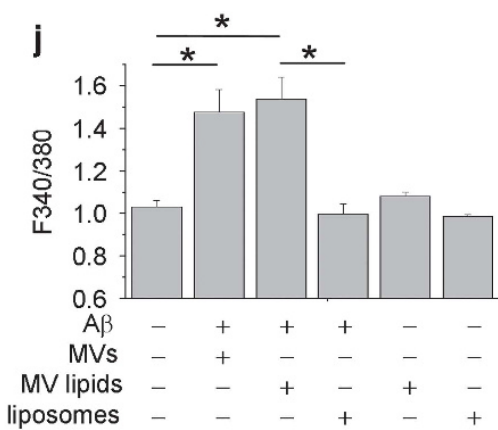

Figure 2 Shed MVs promote formation of soluble forms of $A \beta 1-42$. (a) Basal [ $\left[\mathrm{Ca}^{2+}\right]_{i}$ in neurons exposed for $1 \mathrm{~h}$ either to $\mathrm{A} \beta 1-42 / \mathrm{MV}$ s mixture or soluble (sup)/insoluble (pellet) fractions (left panel, the Kruskal-Wallis ANOVA, $P<0.001$; Dunn's test for comparison among groups, ${ }^{*} P<0.05$ ). Values are normalized to control. Right panel shows the percentage of calcein $-/ \mathrm{PI}+$ neurons under the same conditions (the Kruskal-Wallis ANOVA, $P=0.002$; Dunn's test for comparison among groups, ${ }^{*} P<0.05$ ). (b) Basal $\left[\mathrm{Ca}^{2+}\right]_{i}$ in neurons exposed for $1 \mathrm{~h}$ to MVs derived from resting, M1 or M2 microglia pre-incubated with extracellular A $\beta 1-42$. Values are normalized to control (the Kruskal-Wallis ANOVA, $P<0.001$; Dunn's test for comparison among groups, ${ }^{~} P<0.05$ ). (c) Basal $\left[\mathrm{Ca}^{2+}\right]_{\text {in }}$ neurons exposed to $A \beta 1-42$ and/or MVs in the presence or in the absence of neutralizing antibodies for IL-1 $\beta$ and TNF $\alpha$. Values are normalized to control. (d) Representative ThT fluorescence emission spectra of samples containing A $\beta 1-42$ fibrils (dashed red lines) or aggregated A $\beta$ 1-42 (dashed blue lines) exposed to MVs. (e) Time course of fibrilization of A $\beta$ 1-42 in the presence (dashed line) or in the absence (solid line) of MVs. (f) Negative staining electron microscopic image of aggregated A $\beta 1-42$, incubated overnight in neuronal medium. (g) Representative confocal images of Hylite-488-A $\beta$ 1-42 (488-A $\beta$ 1-42) fibrils untreated or treated overnight with MVs and exposed for $1 \mathrm{~h}$ to neurons. Neurons are stained in blue for MAP-2 after fixation. Cumulative distribution of fibril size from control (solid line) and MV-treated (dashed line) 488-A $\beta 1-42$ fibril preparations is shown on the right. (h) 'Floating assay' by ultracentrifugation reveals an increase of soluble $A \beta 1-42$ species in association with MVs. After centrifugation for $1 \mathrm{~h}$ at $100000 \times g$, MVs are expected in the top fraction, whereas $A \beta$ 1-42 aggregates are expected in the pellet. As ELISA indicates, a higher fraction of A $1-42$ species is transported from the bottom to the top of the gradient in samples incubated overnight with MVs. Acute addition of MVs (AA-MVs) does not cause statistically significant changes in A $\beta 1-42$ distribution (the Kruskal-Wallis ANOVA, $P<0.001$; Tukey's test for comparison among groups, ${ }^{*} P<0.05$ ). (i) ThT fluorescence emission spectra of aggregated A $\beta$ 1-42 (blue lines) or A $\beta$ 1-42 fibrils (red lines), untreated (solid lines) or pretreated (dashed lines) with shed MVs. Spectra of aggregated A $\beta$ 1-42 or A $\beta$ 1-42 fibrils exposed to MVs lipids (dotted lines) are also shown. (j) Basal $\left[\mathrm{Ca}^{2+}\right]_{\text {; }}$ of neurons exposed for $1 \mathrm{~h}$ to $\mathrm{A} \beta 1-42$ pretreated with intact MVs, small unilamellar vesicles of MV lipids (MV lipids) or artificial liposomes. Note that vesicles made by lipids extracted from shed MVs but not artificial liposomes significantly enhance basal $\left[\mathrm{Ca}^{2+}\right]_{i}$ 
fibrils were retrieved in the pellet (Supplementary Figure S1A), whereas globular structures were mostly observed in the supernatant (Supplementary Figure S1B). Detection of globular species in the soluble fraction from $\mathrm{A} \beta$ 1-42 and MVs mixture prompted us to investigate by an array of techniques whether shed MVs change the equilibrium between soluble and insoluble A $\beta$ 1-42.

Possible alterations in the content of aggregated $\mathrm{A} \beta$ 1-42 were first monitored using a thioflavin-T (ThT) dye-binding assay. By this approach, we found that overnight pretreatment with shed MVs caused a $21.26 \pm 0.56 \%$ reduction in fibrillar $A \beta$ $1-42$ (Figure $2 \mathrm{~d}$, red lines; $n=3$ ) and a $38 \pm 6.3 \%$ reduction of aggregated $\mathrm{A} \beta$ 1-42, dissolved in DMSO and incubated overnight at $37^{\circ} \mathrm{C}$ in neuronal medium (Figure $2 \mathrm{~d}$, blue lines; $n=4)$. No changes in ThT spectra were detected upon acute MV addition, thus excluding possible interference of MV lipids with the ThT-binding site of A $\beta 1-42$ (Supplementary Figure S2). Time course analysis of aggregated $A \beta 1-42$ (Figure 2f) confirmed that shed MVs induce aggregate solubilization (Figure 2e). Consistent with these data, confocal microscopy using fluorescently labeled $A \beta$ 1-42 fibrils showed that MVs reduce fibril size (Figure $2 \mathrm{~g}$ ), as indicated by the shift of fluorescent fibrils toward smaller size in the cumulative distribution (Figure $2 \mathrm{~g}$, right $n=3$ ). Altogether, these observations indicate that aggregated $\mathrm{A} \beta$ 1-42 disassembles into soluble species upon MV exposure.

To prove the capability of shed MVs to promote formation of soluble species, soluble amyloid forms generated in A $\beta$ 1-42/ MVs mixture were separated from insoluble species by sucrose gradient centrifugation at $100000 \times g$ for $1 \mathrm{~h}^{13}$ and quantified by ELISA. While acute addition of MVs, immediately before ultracentrifugation on sucrose gradient, partially but not significantly promoted $\mathrm{A} \beta$ 1-42 flotation (AA-MVs, Figure $2 \mathrm{~h}$ ), a marked redistribution of $A \beta 1-42$ to the top of the gradient was detected upon overnight incubation with MVs (Figure 2h; $n=3$ ).

MV lipids promote extracellular formation of neurotoxic A $\boldsymbol{\beta}$ 1-42 species. The next step was to investigate whether lipids were the active components of shed MVs, responsible for the dissolution of insoluble A $\beta 1-42$ species. The ThT assay showed that the lipid fraction extracted from MVs reduced the amount of fibrillar ( $\mathrm{f}-\mathrm{A} \beta$, red lines) or aggregated (A $\beta$, blue lines) $\mathrm{A} \beta 1-42$, similar to intact MVs (Figure $2 \mathrm{i}$ ). Furthermore, assessment of neuron viability revealed a similar percentage of dying neurons, characterized by abnormally high calcium levels, in cultures exposed to $\mathrm{A} \beta$ 1-42 in combination with intact MVs or their lipid component (MV lipids, Figure 2j, $n=3$ ). Notably, A $\beta 1-42$ pre-incubated with synthetic liposomes, similar in size to MVs and mimicking the phospholipid composition of the plasma membrane $(60 \%$ PC, $20 \%$ cholesterol, 10\% SM and 10\% PS) did not produce any increase in the basal calcium concentration (Figure 2j).

Binding of newly generated soluble $A \beta$ 1-42-488 to neurons is competed by $\mathrm{PrP}^{\mathrm{c}}$. We next attempted to visualize soluble $A \beta$ 1-42 forms, generated in the presence of MVs, by imaging their binding to cultured neurons. We observed that culture exposure to $488-\mathrm{A} \beta \quad 1-42 / \mathrm{MVs}$ mixture for $1 \mathrm{~h}$ produced a strong labeling of MAP-2-positive dendrites, which exceeded by almost fourfolds the staining produced by fluorescent $A \beta 1-42$ alone (Figures $3 a$ and $b$ ). 488-A $\beta 1-42$ binding to dendrites was paralleled by a marked reduction of MAP-2 staining (Figure 3a), according to previous evidence. ${ }^{36}$ No preferential association of 488-A $\beta 1-42$ with synapses was detected (data not shown). Fractionation of $488-\mathrm{A} \beta \quad 1-42 / \mathrm{MVs}$ mixture into two phases by centrifugation showed that the fluorescent $A \beta 1-42$ forms capable of binding to neurons were mainly recovered in the soluble fraction (Supplementary Figures S1C-E). Notably, $\mathrm{A} \beta$ binding was specifically competed by the high-affinity oligomer-interacting protein $\mathrm{PrP}^{\mathrm{c} 16}$ and virtually abolished by the anti-A $\beta$ antibodies $\mathrm{A}_{11}$ and $6 \mathrm{E} 10$ (Figures $3 a$ and $\mathrm{b}$ ) Unlike the 89-230 truncated $\operatorname{PrP}^{\mathrm{C}}$, both full-length folded $\mathrm{PrP}^{\mathrm{C}}$ and anti-A $\beta$ Abs neutralized the toxicity of $\mathrm{A} \beta 1-42 / \mathrm{MVs}$ mixture, as revealed by calcium recording (Figure $3 c ; n=3$ ) and $\mathrm{Pl} /$ calcein staining (Figure $3 \mathrm{~d} ; n=3$ ).

Finally, as the soluble but not the fibrillar $\mathrm{A} \beta$ forms activate NMDA receptors, ${ }^{37}$ we used a bioassay to assess the capability of $\mathrm{A} \beta$ species generated in the presence of MVs to enhance NMDA receptor activity. FURA-2-loaded neurons, expressing functional NMDA receptors, were used as sensor cells for soluble $A \beta 1-42$. By this approach, we detected calcium responses in about $30 \%$ of neurons exposed to $A \beta 1-$ 42 in combination with MVs, but not $A \beta 1-42$ or shed MVs alone (Figure $3 e ; n=3$ ). No calcium transients were observed upon neuron challenging with scrambled $\mathrm{A} \beta$ 1-42 and MVs (data not shown). Calcium responses evoked by A $\beta$ 1-42 pretreated with MVs were inhibited by the NMDA receptor antagonist APV $(100 \mu \mathrm{M})$ (Figures $3 f$ and $g ; n=3)$. Direct HPLC measurements of glutamate content in MVs or $\mathrm{A} \beta 1-$ $42 / \mathrm{MVs}$ preparations revealed concentrations lower than $1 \mu \mathrm{M}$, that is, the minimal concentration required to detect calcium influx in our system (mean glutamate concentration: $137 \pm 70 \mathrm{nM}$, MVs alone; $196 \pm 115 \mathrm{nM}, \mathrm{A} \beta 1-42 / \mathrm{MVs}$ mixtures). These data exclude possible interference of ambient glutamate in the NMDA-dependent calcium response. Therefore, soluble $\mathrm{A} \beta$ 1-42 species generated in the presence of shed MVs are able to activate NMDA calcium channels, triggering excitotoxicity.

MVs carry neurotoxic species generated from internalized A $\boldsymbol{\beta}$ 1-42. As amyloid plaques are surrounded by activated microglia that actively phagocyte and degrade $\mathrm{A} \beta$, we investigated whether MVs may contain toxic $A \beta$ species, generated from internalized peptides. Confocal analysis of microglia exposed to $\mathrm{A} \beta 1-42$ for $24-48 \mathrm{~h}$, extensively washed and stained with $6 \mathrm{E} 10$ anti-A $\beta$ antibody, revealed intracellular $\mathrm{A} \beta$ aggregates, which can reach the plasma membrane, stained by IB4 (Figure 4a). Notably, few A $\beta$ and IB4 double-positive particles were detected extracellularly in cell proximity (Figure $4 a$, bottom right panel), suggesting that EMVs derived from $\mathrm{A} \beta$-loaded microglia may indeed contain $\mathrm{A} \beta$ species. We explored this hypothesis by western blot analysis of EMVs collected from supernatant of microglia exposed to biotynilated A $\beta 1-42$. Upon ATP stimulation for $30 \mathrm{~min}$, a condition that mimics an inflammatory context and favors shedding of MVs (P2 and P3 fraction) versus exosome (P4 fraction) release $^{38}$ biotin-conjugated $\mathrm{A} \beta 1-42$ was 

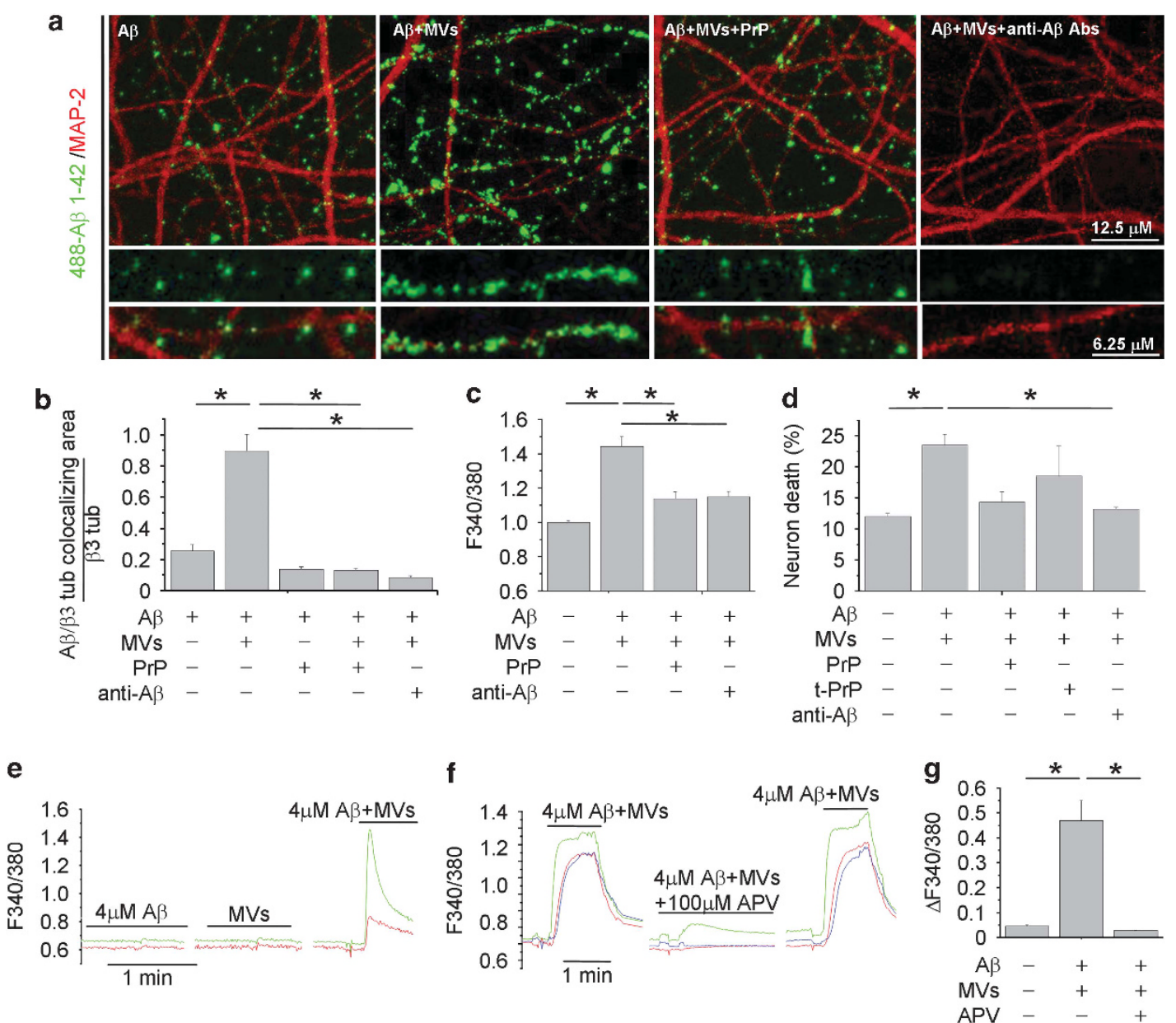

Figure 3 Binding of newly generated soluble 488-A $\beta-1-42$ to neurons is competed by $\operatorname{PrP}^{\mathrm{C}}$. (a) Representative confocal images of 14DIV neurons exposed to 488-A $\beta$ 1-42 alone or in combination with MVs, with or without pretreatment with PrP or with the anti-A $\beta$ antibodies $A 11$ and $6 E 10$. (b) Corresponding quantification of 488-A $\beta$ 1-42 binding to cultured neurons expressed as colocalizing area between 488-A $\beta$ and $\beta$ tubulin, relative to total $\beta$ tubulin (see Materials and Methods) (the Kruskal-Wallis ANOVA, $P<0.001$; Dunn's test comparison among groups, ${ }^{*} P<0.05$ ). (c and d) Basal $\left[\mathrm{Ca}^{2+}\right]_{i}$ (c) and percentage of calcein - $/ \mathrm{PI}+$ neurons (d) in 9-14 DIV cultures exposed to different combinations of $A \beta 1-42, \mathrm{MVs}$, A11 plus $6 \mathrm{E} 10$ antibodies, full-length or truncated (tPrPC) $\operatorname{PrP}^{\mathrm{C}}$ (the Kruskal-Wallis ANOVA, $P<0.001$; Dunn's test for comparison among groups, $\left.{ }^{*} P<0.05\right)$. (e-g) Bio-detection of soluble A $\beta 1-42$ by fura-2-loaded sensor neurons, expressing functional NMDA receptors. Representative traces of $\left[C a^{2+}{ }_{i}\right.$ changes recorded in neurons upon exposure to $\mathrm{KRH}$ containing $\mathrm{A} \beta 1-42$ alone $(4 \mu \mathrm{M})$ or MVs alone $(1 \mu \mathrm{g} / 100 \mu \mathrm{l})$ or their combination (e). [Ca $\left.{ }^{2+}\right]_{i}$ responses induced by $\mathrm{A} \beta$ $1-42 / M V s$ mixture are strongly inhibited by the NMDA receptor antagonist APV (f), as quantified in $\mathbf{g}$. Values represent peak $\left[\mathrm{Ca}^{2+}\right]_{i}$ increases $(\Delta \mathrm{F} 340 / 380$ fluorescence) from about 30 neurons/condition (the Kruskal-Wallis ANOVA, $P<0.001$; Dunn's test for comparison among groups, ${ }^{*} P<0.05$ )

recovered in shed MVs, labeled by Tsg101 (Figure 4b, bottom panel). Consistently, SELDI-TOF mass spectrometry using $6 \mathrm{E} 10$ and $4 \mathrm{G} 8$ anti-A $\beta$ antibodies revealed the presence of preloaded $A \beta 1-42$ and of its cleavage product A $\beta 1-40$, along with traces of other carboxy-terminally truncated isoforms, in MVs shed from the plasma membrane (P2 + P3 fractions) (Figure $4 \mathrm{c}$ ). Ten times less $\mathrm{A} \beta$ was recovered in exosomes (P4 fraction) (Figure 4c), although A $\beta 1-42$ species were clearly detectable in exosomes constitutively accumulated for $24 \mathrm{~h}$ in microglia supernatant (Supplementary Figure S3). In line with the presence of $A \beta$ species, MVs derived from microglia stimulated for $48 \mathrm{~h}$ with $4 \mu \mathrm{M} \mathrm{A} \beta$ 1-42 (A $\beta$-MVs) were highly neurotoxic as compared with MVs derived from resting cells (Figure $4 d, n=4$ ). MV neurotoxicity was significantly decreased by anti-A $\beta$ antibodies. These data indicate that microglia internalize and generate $\mathrm{A} \beta$ neurotoxic species, which are delivered to neurons in association with MVs, possibly on MV external membrane.

MVs from AD patients are neurotoxic. Recent results from our laboratories indicated that microglia-derived MVs are detectable in the CSF of humans and that their amount reflects the extent of microglia activation in the course of neuroinflammation. ${ }^{30}$ To verify whether production of MVs from microglia could be elevated in $A D$, we collected CSF from patients with mild cognitive impairment $(\mathrm{MCl})$ or $A D$, as well as from age- and gender-matched healthy donors. Flow cytometry analysis showed strikingly higher levels of MVs positive for the myeloid marker IB4 (more than 10-fold) in $\mathrm{MCl}$ and $\mathrm{AD}$ patients than in control subjects (Figure $5 \mathrm{a}$ ). IB4-positive MVs accounted for $\sim 65 \%$ of total EMVs detectable by flow cytometry. The number of IB4-positive MVs is correlated with a known CSF marker of 


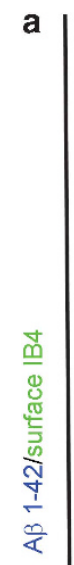
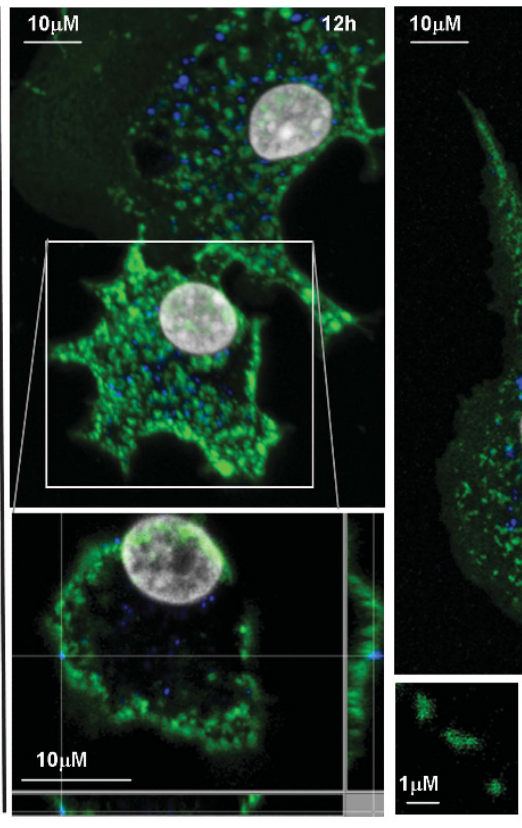

4000 4500

d

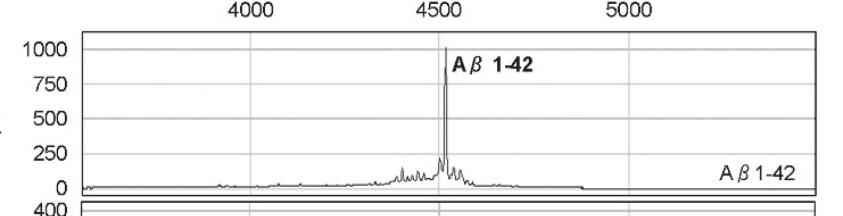

$\S$

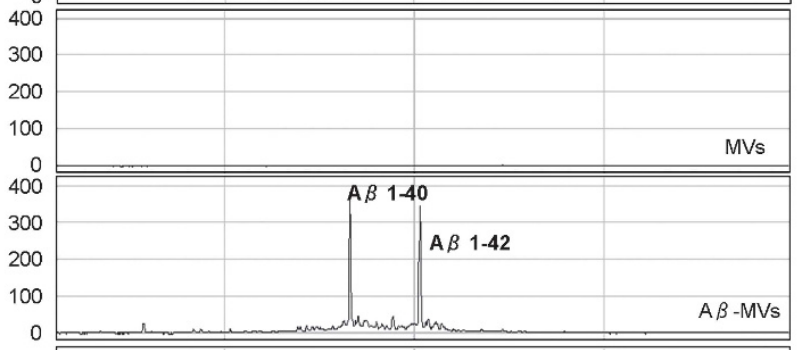

$\S$
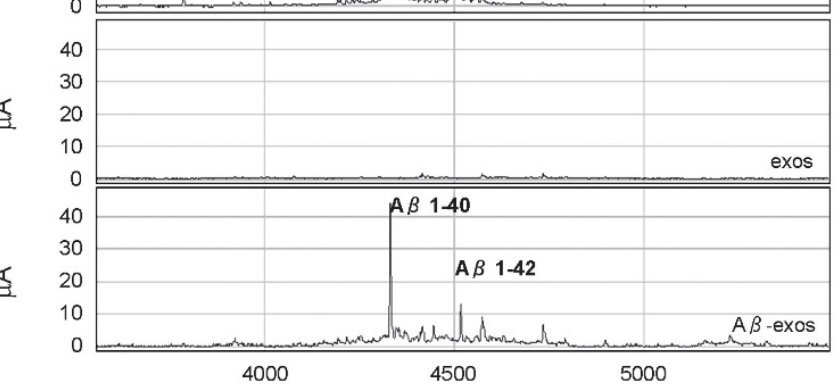

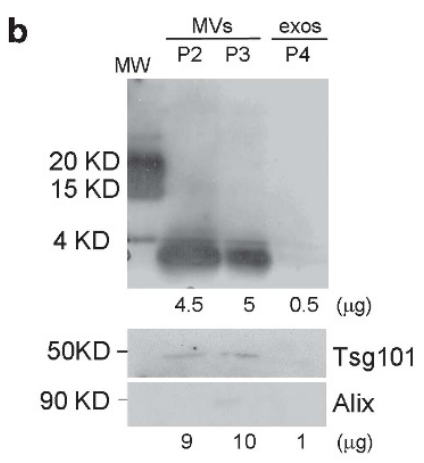

C

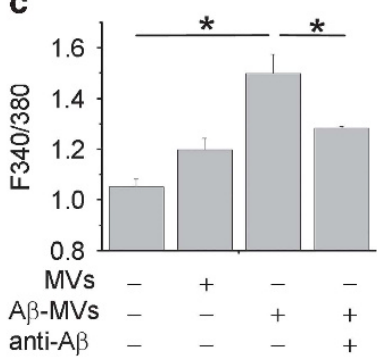

Figure 4 Soluble $A \beta$ forms are released in association with shed MVs from microglia activated with $A \beta 1-42$. (a) Living rat microglia were exposed to human $A \beta 1-42$ for $12-48 \mathrm{~h}$ and stained with IB4-FITC to label the cell surface before being fixed and counterstained with 6 E10 antibody, which recognizes human but not rat amyloids. Top left panel shows representative xy plane maximum projection of microglia, revealing several $6 \mathrm{E} 10$ immunoreactive puncta inside the cells, some of which are double positive for surface IB4-FITC. Bottom left panel showing single stack of the selected cell, shown at higher magnification, reveals a clear association of internalized A $\beta$ 1-42 to the cell surface, further revealed by the $z$ axis scan. Note, an increase in the size of internalized $A \beta 1-42$ after incubation for $48 \mathrm{~h}$ (top right panel). Examples of EMVs, double positive for 6 E10 and IB4-FITC are shown in bottom right panels. (b) Western blot analysis of A $\beta 1-42$ species present in shed MVs (P2 and P3 fractions) and exosomes (P4 fraction) released upon $30 \mathrm{~min}$ ATP stimulation by $4 \times 10^{6}$ microglia pre-exposed to biotinylated A $\beta 1-42(4 \mu \mathrm{M})$. Blots were carried out using a $15 \%$ Tris-glycine gel and membranes were probed with streptavidin. Shed MVs and exosomes produced by $8 \times 10^{6}$ donor microglia were probed in parallel for the EMV markers Tsg101 and the exosomal marker Alix (lower panels). Numbers below each lane indicate the estimated amount of loaded proteins. (c) Shed MVs and exosomes produced by $1 \times 10^{6}$ rat microglia pre-exposed to human A $\beta$ 1-42 were analyzed by a SELDI-TOF MS immunoproteomic assay employing anti-human A $\beta$ antibodies (4G8 and 6 E10) on PS20 chip array to capture A $\beta 1-42$ and carboxy-terminally truncated $\mathrm{A} \beta$ isoforms. The following representative spectra of samples in NP40 1\% lysis buffer are shown (from top to bottom): $4 \mu \mathrm{M} \mathrm{A} \beta 1-42$ peptide incubated overnight in KRH; MVs from control microglia, not exposed to A $\beta 1-42$; MVs from A $\beta 1-42$ preloaded microglia (A $\beta$-MVs); exosomes from control microglia (exos);

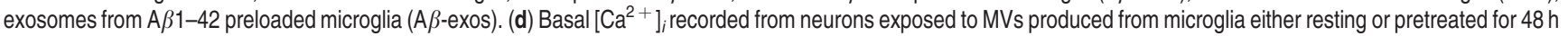
with $\mathrm{A} \beta$ 1-42 (A $\beta$-MVs), in the presence or in the absence of anti-A $\beta$ antibodies (A11 +6E10) (the Kruskal-Wallis ANOVA, $P<0.001$; Dunn's test for comparison among groups, $\left.{ }^{*} P<0.05\right)$. See also Supplementary Figure $\mathrm{S} 1$ 


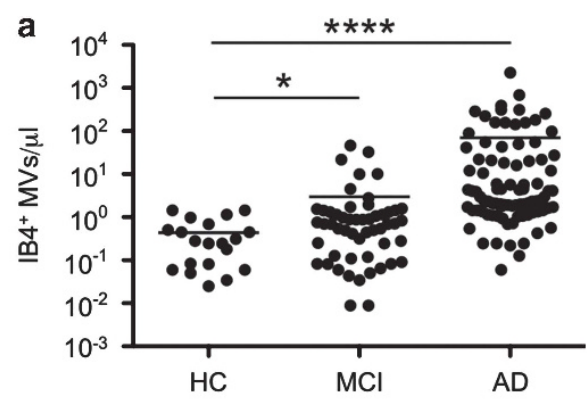

C
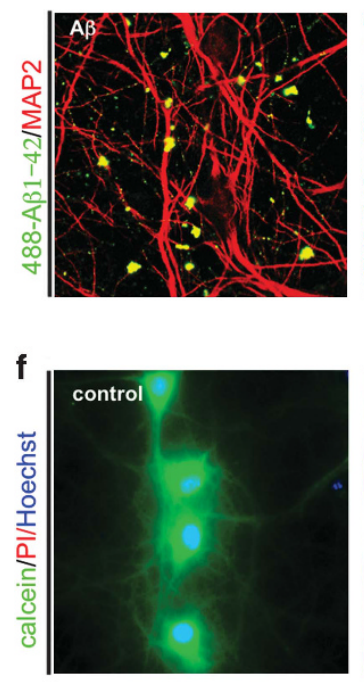
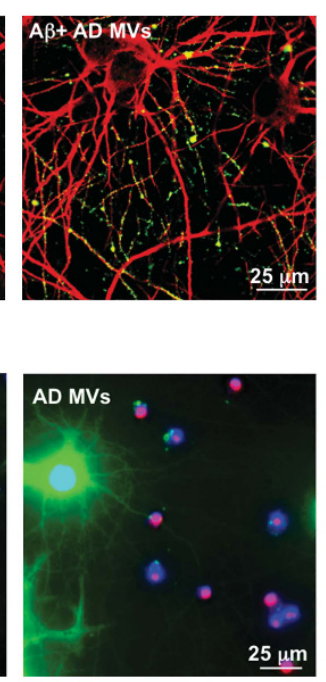

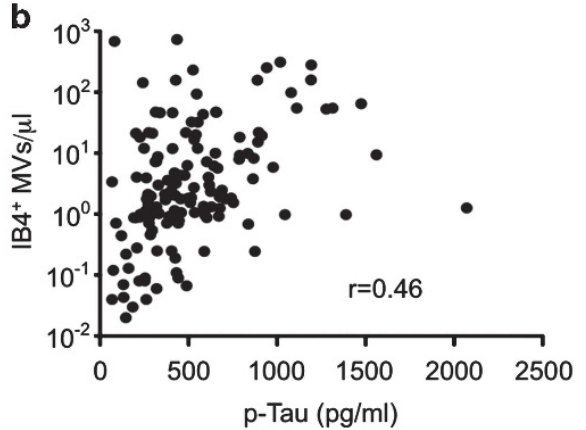

d

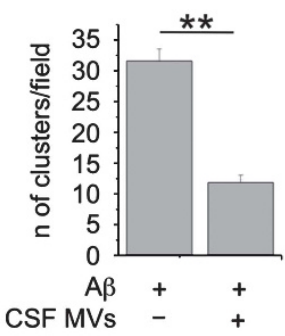

g

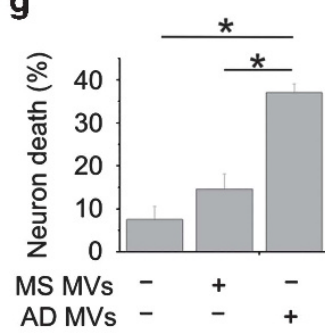

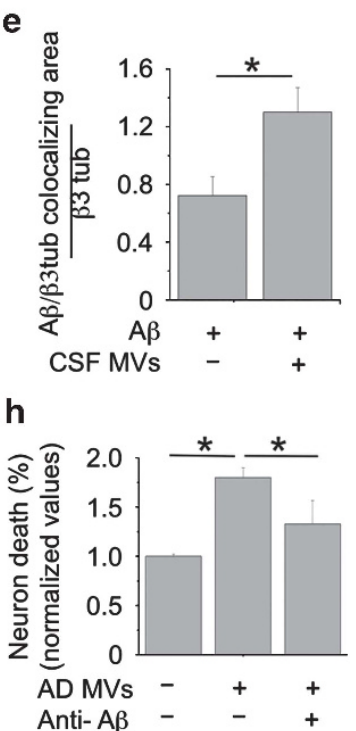

Figure 5 CSF MVs isolated from AD patients. (a) Quantitative flow cytometry analysis of IB4 ${ }^{+}$MVs in CSF collected from MCI patients $(n=53)$, AD patients $(n=89)$ and age- and gender-matched controls ( $\mathrm{HC} ; n=20$ ) (the Mann-Whitney test, $P<0.0001 \mathrm{AD}$ versus $\mathrm{HC} ; P<0.0329 \mathrm{MCl}$ versus $\mathrm{HC}$ ). (b) Correlation between IB4 ${ }^{+} \mathrm{MV}$ s and total tau protein in the CSF of $\mathrm{MCl}$ and $\mathrm{AD}$ patients, (rho $=0.46, P<0.0001$, Spearman's correlation). (c) Representative confocal images of cultured neurons, exposed to aggregated 488-A $\beta$ 1-42 untreated or pretreated overnight with MVs from AD patients and stained for MAP-2 after fixation (red). A $\beta$ species bind to MAP-2 dendrites. Note the decrease in the number of large fluorescent $A \beta$ clusters in neurons exposed to 488-A $\beta$ 1-42 in combination with AD MVs. (d) Quantification of 488-A $\beta$ 1-42 aggregates (larger than $5 \mu \mathrm{m}$ ) per field (data follow normal distribution, Student's $t$-test, ${ }^{* *} P<0.001$ ). (e) Quantification of 488-A $\beta 1-42$ binding to cultured neurons, expressed as colocalizing area between $488-\mathrm{A} \beta$ and $\beta$ tubulin, relative to total $\beta$ tubulin (see materials and Methods; data follow normal distribution, Student's $t$-test, ${ }^{* *} P<0.001$ ). (f) Representative fluorescence microscopy images of 14DIV neurons triple stained for calcein, $\mathrm{PI}$ and Hoechst $24 \mathrm{~h}$ after exposure to AD MVs or maintained in control conditions. (g) Quantification of the percentage of calcein - $/ \mathrm{PI}+$ neurons (dead cells) in cultures exposed to AD MVs or MVs isolated from patients with multiple sclerosis (the Kruskal-Wallis ANOVA, $P<0.001$; Dunn's test for comparison among groups, ${ }^{*} P<0.05$ ). (h) Percentage of dead neurons in cultures exposed to MVs isolated from the CSF of AD patients in the presence of anti-A $\beta$ antibodies A11 and 6 E10 (ANOVA, $P<0.001$, the Holm-Sidak method, $P<0.05$ ). See also Supplementary Figure S2 and Supplementary Table S1

neurodegeneration, namely, total Tau protein (Figure 5b; $P<0.0001){ }^{39}$ We next examined the effects of MVs collected from AD patients on the equilibrium between soluble and insoluble $\mathrm{A} \beta$ 1-42 species and assessed their toxic potential on cultured neurons. Confocal analysis of neurons exposed to 488-A $\beta$ 1-42 pre-incubated overnight with $A D M V s$ revealed a threefold decrease in the content of fluorescent $A \beta$ aggregates (Figures $5 c$ and $d$ ) and a parallel increase in fluorescent $A \beta$ species bound to dendrites (Figures $5 c$ and e).

Consistently, with in vitro results showing that microglial MVs carry neurotoxic species generated from internalized $\mathrm{A} \beta$ 1-42, $M V s$ recovered from $A D$ patients were highly toxic, as indicated by quantification of calcein - /PI + dead neurons, as compared with MVs isolated from patients with multiple sclerosis (Figures $5 f$ and $g, n=3$ ). Neurotoxicity of $A D$ MVs was significantly decreased by pretreatment with anti-A $\beta$ antibodies (Figure $5 \mathrm{~h}$ ).
Interestingly, $\mathrm{A} \beta$ 1-40, $\mathrm{A} \beta$ 1-42 and other truncated $\mathrm{A} \beta$ peptides were detected by SELDI-TOF mass spectrometry in CSF MVs of a patient affected by AD (Supplementary Figure S4).

\section{Discussion}

In the present study, we unveil a novel mechanism by which microglia contribute to neuronal damage in AD. We show that MVs, extracellularly released by cultured microglia, strongly increase $\mathrm{A} \beta$ neurotoxicity in vitro. This effect is due to the lipid components of MVs, which promote formation of small soluble neurotoxic species from $\mathrm{A} \beta$ 1-42 extracellular aggregates. Although $\mathrm{A} \beta$ species can associate with MVs, as suggested by increased $\mathrm{A} \beta$ flotation on sucrose gradient upon acute addition of MVs, most of neurotoxic soluble forms do not bind to MVs strongly. This is indicated by our observations that free 
soluble A $\beta$ 1-42 species, not associated with MVs, bind efficiently to a subpopulation of neurons in vitro, increase NMDA receptor permeability and cause an excitotoxic damage. Conversely, A $\beta$ 1-42 forms pelleted along with MVs from $A \beta / M V s$ mixture display low toxicity and little neuronal-binding capacity. These data identify microglial MVs as an endogenous source of lipids able to shift the equilibrium toward toxic $\mathrm{A} \beta$ species. This conclusion is in complete agreement with previous evidence that brain membrane lipids, including phosho- and (glycol)sphingolipids, favor formation of soluble forms, either promoting solubilization of inert fibrils, ${ }^{13}$ or hindering their conversion to insoluble fibrils. ${ }^{14}$ Interestingly, exosomes released by neurons have been found to promote rather than reduce $A \beta$ fibrillogenesis, ${ }^{40}$ thus indicating that lipid composition of different EMVs generated by distinct cell types may have opposite effects on $\mathrm{A} \beta$ extracellular assembly. Notably, MVs have a distinct repertoire of lipids not only compared with exosomes (our unpublished data) ${ }^{41}$ but also to the plasma membrane of origin. Indeed, MVs are enriched in cholesterol, sphingomyelin and ceramide, and contain lipid raft elements, ${ }^{28}$ including GM1 and GM3 gangliosides and flotillin-2. ${ }^{42}$ Accordingly, artificial liposomes, composed of the main phospholipids of the plasma membrane, neither induce fibril solubilization nor promote $\mathrm{A} \beta$ neurotoxicity. Lipidomic profiling of microglial MVs will identify endogenous lipids responsible for the generation of neurotoxic $A \beta$ species, which could themselves represent putative $A D$ biomarkers. $9,43,44$

MV-mediated $A \beta$ processing, leading to neurotoxic forms does not occur only extracellularly. Indeed, microglial MVs also contain toxic forms generated from internalized $\mathrm{A} \beta$ 1-42. It has been previously shown that a fraction of intracellular $\mathrm{A} \beta$ can be released through exosomes by neurons and oligodendrocytes. $^{32,45-47}$ In addition, phagocytosed $\mathrm{A} \beta$ has been found to be re-secreted from microglia, although through an undefined mechanism. ${ }^{48}$ We now show that microglia release neurotoxic $\mathrm{A} \beta 1-42$ and $\mathrm{A} \beta 1-40$ species in association with MVs. This is the first evidence that microglia - which control extracellular plaque load ${ }^{4,50}$ by phagocytosis and degradation of $\mathrm{A} \beta$ fibrils or macropinocytosis of soluble $\mathrm{A} \beta^{51,52}$ - may seed and feed formation of neurotoxic amyloids throughout the brain. MV-mediated release of neurotoxic $A \beta$ forms likely occurs when intracellular pathways of $\mathrm{A} \beta$ degradation are saturated and production of MVs becomes a way for microglia to eliminate undigested $\mathrm{A} \beta$. Neurotoxic $\mathrm{A} \beta$ species may be processed in early to late endosomes and lysosomes ${ }^{53}$ after disassembly of phagocytosed $\mathrm{A} \beta$ and sorted to the external surface of MVs through association with the GPI-anchored protein $\mathrm{PrP}^{\mathrm{C}}$ or $\mathrm{GM} 1$ gangliosides, all of which are localized to raft domains ${ }^{42}$ and bind tightly $A \beta$ oligomers. ${ }^{54}$ Alternatively, neurotoxic $A \beta$ species may be generated at the cell surface, ${ }^{55}$ where components of the $\gamma$-secretase complex, which can cleave the carboxyl terminal of $A \beta 1-42$ at position 40 are also localized, ${ }^{53}$ possibly inside lipid rafts. This sorting mechanism may be consistent with the proposed role of lipid rafts in setting up platforms to concentrate into MVs proteins destined to secretion. ${ }^{28,56}$ Finally, processing of $A \beta 1-42$ to $A \beta 1-40$ may even proceed within MVs. Indeed, previous evidence showed that neuron-derived EMVs contain some components of the $\gamma$ secretase complex, ${ }^{57}$ whereas the insulin-degrading enzyme, which proteolyzes $A \beta 1-42$ and $A \beta 1-40$, has been detected among cargo proteins of microglial EMVs. ${ }^{58}$ The significant decrease in neurotoxicity observed upon pretreatment with anti-A $\beta$ antibodies strongly supports the theory that neurotoxic $\mathrm{A} \beta$ forms are in fact localized to the outer lipid bilayer of MVs. However, further studies are required to unequivocally define the topology of $\mathrm{A} \beta$ species and to clarify whether $\mathrm{A} \beta$ forms are actually associated to the extracellular membrane of shed MVs.

Our findings have clear clinical implications. First, production of $\mathrm{MVs}$ is very high in $\mathrm{MCl}$ and $\mathrm{AD}$ patients, reflecting microgliosis, ${ }^{30}$ which typically characterizes the disease. ${ }^{8}$ Second, MVs collected from the CSF of AD patients promote extracellular formation of neurotoxic $A \beta$ species similar to MVs shed from cultured cells. Finally, MVs collected from AD patients are extremely toxic for cultured neurons and their neurotoxicity results, at least in part, from their $\mathrm{A} \beta$ cargo. However, it is still to be defined whether MVs may associate with toxic forms of $\mathrm{A} \beta$ present in the parenchyma/blood vessel as well as plaques during their travel to CSF. In agreement with their pathogenic role, levels of microglia-derived MVs are positively correlated with classical biomarkers of neuronal injury such as tau ${ }^{39}$ in $\mathrm{MCl}$ and $\mathrm{AD}$ subjects, and with damage to white matter structures of the temporal lobe in $\mathrm{MCl}$ patients, as revealed by MRI scans (Dalla Libera et al., manuscript in preparation). Correlation between microglia-derived MVs and brain damage suggests that MVs may represent a novel companion tool for AD diagnosis, and paves the way for future therapies targeting MVs to control the impact of neurotoxic $A \beta$ species on brain function. We anticipate that analysis of $A \beta$ content and lipidomic profiling of MVs in a large cohort of AD and healthy subjects will clarify whether changes in the conformation and/or in the amount of $A \beta$ forms account for MV neurotoxicity. Furthermore, lipidomic profiling of human MVs will lead to the identification of new putative AD biomarkers, thereby increasing the diagnostic potential of MVs in AD.

\section{Materials and Methods}

Glial cells and MVs isolation. Primary rat microglial cells were isolated from mixed cultures of cortical and hippocampal astrocytes, established from E21 rat embryos and maintained as described previously. ${ }^{27}$ All efforts were made to minimize animal suffering and to reduce the number of animals used in accordance with the European Communities Council Directive of September 20, 2010 (2010/63/UE). All procedures involving animals were performed according to the guidelines of the Institutional Animal Care and Use Committee of the University of Milan.

To induce MV shedding, microglia were exposed to ATP (1 mM) for $30 \mathrm{~min}$ in Krebs-Ringer solution $(\mathrm{KRH})$. Shed MVs were pelletted from the supernatant at $10000 \times g$ for $30 \mathrm{~min}$, whereas exosomes were pelleted at $100000 \times g$ for $1 \mathrm{~h}$, as described previously. ${ }^{38}$ For biochemical fractionation of shed MVs, total lipids were extracted through the method previously described ${ }^{59}$ with $2: 1$ (by volume) of chloroform and methanol. The lipid fraction was evaporated under a nitrogen stream, dried and resuspended in PBS at $40^{\circ} \mathrm{C}$ in order to obtain multilamellar vesicles. Small unilamellar vesicles were obtained by sonicating multilamellar vesicles.

A $\beta$ 1-42 preparations. Unless otherwise stated, A $\beta$ 1-42 (Anaspec, Fremont, CA, USA) was dissolved at a concentration of $2 \mathrm{mM}$ in DMSO. The stock was kept at $-80^{\circ} \mathrm{C}$, directly diluted to $4 \mu \mathrm{M}$ in neuronal medium and kept overnight at $37^{\circ} \mathrm{C}$. We refer to this $\mathrm{A} \beta 1-42$ preparation as aggregated $\mathrm{A} \beta$ $1-42$, to distinguish it from fibrillar $A \beta 1-42$ preparation, which is detailed below. To prepare soluble and fibrillar $\mathrm{A} \beta 1-42$, the peptide was initially monomerized by dissolving it in $100 \%$ hexafluoroisopropanol (Sigma, St. Louis, MO, USA) to obtain a 1-mM solution and then aliquoted in sterile microcentrifuge tubes. 
The hexafluoroisopropanol was removed under vacuum using a SpeedVac and the peptide film was stored (desiccated) at $-80^{\circ} \mathrm{C}$. Soluble $A \beta$ 1-42 was prepared as described by Klein et al. ${ }^{60}$ Briefly, the peptide film was freshly resuspended in $100 \%$ DMSO to $5 \mathrm{mM}$, further diluted to $100 \mu \mathrm{M}$ in $\mathrm{F}-12$ medium (Invitrogen, Paisley PA4 9RF, UK) and incubated for $24 \mathrm{~h}$ at $5{ }^{\circ} \mathrm{C}$. Following incubation it was centrifuged at $14000 \times \mathrm{g}$ for $10 \mathrm{~min}$ at $4{ }^{\circ} \mathrm{C}$ and the soluble forms were collected in the supernatant. For fibrillar $A \beta 1-42$ preparation, $A \beta 1-42$ or Hylite-488-A $\beta$ 1-42 peptide film freshly resuspended in DMSO was further diluted to $100 \mu \mathrm{M}$ in $10 \mathrm{mM} \mathrm{HCl} .{ }^{61}$ It was vortexed for $15 \mathrm{~s}$ and incubated for $24 \mathrm{~h}$ at $37^{\circ} \mathrm{C}$. After incubation, it was diluted to $4 \mu \mathrm{M}$ in neuronal medium.

The aggregation state of $A \beta 1-42$ preparation was assessed by transmission electron microscopy with a Tecnai G2 T20 Twin microscope (FEl, Eindhoven The Netherlands)

ThT assay. $\mathrm{A} \beta$ preparations, incubated or not with MVs, were diluted to $4 \mu \mathrm{M}$ in $\mathrm{KRH}$ and incubated overnight at $37^{\circ} \mathrm{C}$. For ThT assays, ThT (Fisher Scientific, Waltham, MA, USA) was added to the $\mathrm{A} \beta$ preparations and monitored in a PerkinElmer LS50 spectrofluorometer (Perkin-Elmer, Waltham, MA, USA). ThT fluorescence emission spectra were recorded between 465 and $565 \mathrm{~nm}$ with $5 \mathrm{~nm}$ slits, using an excitation wavelength of $450 \mathrm{~nm}$. For the time course experiments, the samples were kept at $37^{\circ} \mathrm{C}$ and aliquots of $100 \mu$ were removed from the sample at each time point. The aliquots were mixed with ThT to obtain a final concentration of $10 \mu \mathrm{M}$ and then their fluorescence spectra were acquired.

Neuronal cultures and in vitro stimulation. Primary cultures of hippocampal neurons were established from E18 rat pups as previously described ${ }^{62}$ plated onto poly-L-lysine-treated coverslips at $500 \mathrm{cell} / \mathrm{s} / \mathrm{mm}^{2}$ cell density and maintained in Neurobasal with 2\% B27 supplement and $2 \mathrm{mM}$ glutamine (neuronal medium). DIV hippocampal neurons (9-14) were exposed to $\mathrm{A} \beta 1-42(4 \mu \mathrm{M})$, to MVs $(1 \mu \mathrm{g} / 100 \mu \mathrm{l})$ or to a combination of $\mathrm{A} \beta 1-42$ and MVs for $1 \mathrm{~h} . \mathrm{A} \beta 1-42$, MVs or their combination were kept overnight at $37 \mathrm{C}^{\circ}$ before being exposed to neurons. Neurons $\left(1.7 \times 10^{5}\right)$ were exposed to MVs produced by $1 \times 10^{6}$ microglia (microglia: neuron ratio, $6: 1$ ).

In a set of experiments, $A \beta 1-42 / M V$ s mixtures were added with anti-TNF- $\alpha$ plus antiIL-1 $\beta$ antibodies (R\&D, Minneapolis, MN, USA) or with the anti-A $\beta$ antibodies 6 E10 (Covance, Emerville, CA, USA) plus A11 (Invitrogen, Life Technologies Ltd., Paisley, UK), or with the $\operatorname{PrP}^{C}(4 \mu \mathrm{M})$ for 30 min before being exposed to cultured neurons.

\section{Cell viability assays}

$\mathrm{Pl} /$ calcein staining: Neuron viability was analyzed by simultaneous fluorescence staining of viable and dead cells with calcein-AM $(0.5 \mathrm{mg} / \mathrm{ml}$, Invitrogen, Life Technologies Ltd.), PI (1 $\mu \mathrm{g} / \mathrm{ml}$, Molecular Probes, Life Technologies Ltd., Paisley, UK) and Hoechst (8.1 $\mu \mathrm{M}$, Molecular Probes, Life Technologies Ltd.). Incubation was performed for $20 \mathrm{~min}$ in neuronal medium at $37^{\circ} \mathrm{C}$ and $5 \% \mathrm{CO}_{2}$. Calcein-AM emits green fluorescence signal in viable cells. Conversely, $\mathrm{PI}$ reaches nuclei of dead cells only where it emits red fluorescence. Fluorescence images were acquired by Leica DMI 4000B microscope (Leica Microsystem GmbH, Wetzlar, Germany), equipped with DIC microscopy. The percentage of neuronal death was calculated as the ratio of $\mathrm{PI}+$ calcein-dead cells to the total number of Hoechst stained neurons in at least 15 fields/condition.

Annexin-V assay. Living neurons were incubated with annexin-V-FITC (1:100, BD Pharmingen, Franklin Lakes, NJ, USA) for 5 min, fixed with $4 \%$ paraformaldehyde and counterstained for the neuronal marker SNAP-25 (mouse anti-SNAP-25, Sternberger Monoclonals, Baltimora, MD, USA) in nonpermeabilizing condition to preserve annexin-V staining. Fluorescence images were acquired by a SPE Leica confocal microscope, equipped with an ACS APO $\times 40 / 1.15$ oil objective. Area of annexin-V + apoptotic processes was quantified by Image J 1.46r software $(\mathrm{NIH}$, Bethesda, MD, USA) and normalized to SNAP-25 immunoreactive area as an index of neurite density.

Monitoring of cytoplasmic calcium concentration: Hippocampal neurons were loaded with $2 \mu \mathrm{M}$ Fura-2/AM (Invitrogen, Life Technologies Ltd.) in neuronal medium for $40 \mathrm{~min}$ at $37^{\circ} \mathrm{C}$, washed in $\mathrm{KRH}$ and transferred to the recording chamber of an inverted microscope (Axiovert 100, Zeiss, Oberkochen, Germany) equipped with a calcium imaging unit. Polychrome V (TILL Photonics GmbH, Grafelting, Germany) was used as the light source. Images were collected with a CCD Imago-QE camera (TILL Photonics GmbH) and analyzed with TILLvisION 4.01 software. After excitation at 340 and $380 \mathrm{~nm}$ wavelengths, the emitted light was acquired at $505 \mathrm{~nm}$ at $1 \mathrm{~Hz}$. Calcium concentration was expressed as F340/
380 fluorescence ratio. The ratio values in selected region of interest corresponding to neuronal somata were calculated from sequences of images to obtain temporal analysis. Basal calcium concentration was recorded from at least 100 neurons/condition in each experiment.

Immunocytochemical staining. Immunofluorescence staining of fixed neurons was performed using the following antibodies: rabbit anti-beta tubulin (Sigma) guinea pig anti-vGLUT-1 (Synaptic System, Gottingen, Germany), mouse anti-PSD-95 (UC Davis/NIH NeuroMab Facility, Davis, CA, USA) and mouse antiMAP-2 (Synaptic System). Secondary antibodies were conjugated with Alexa-488, Alexa-555 or Alexa-633 fluorophores (Invitrogen, Life Technologies Ltd.). For quantification of V-glut-1 puncta per length unit, the length of single neuritis was measured using Image $\mathrm{J} 1.46 \mathrm{r}$ software, and the number of positive puncta whose dimension was greater than $0.01 \mu \mathrm{m}$ was quantified.

Binding to neurons of Hylite-488-labeled A $\beta$ 1-42 (Anaspec) was quantified using Image $\mathrm{J} 1.46 \mathrm{r}$ software. Hylite-488-A $\beta \quad 1-42$ and $\beta$ tubulin double-positive puncta were revealed by generating a Hylite- $488-\mathrm{A} \beta 1-42 / \beta$ tubulin double-positive image using the 'and' option of 'image calculator' function. A fixed threshold was then set in the double-positive image and, having selected the pixel area parameter at 3-infinite, total colocalizing area was quantified using the 'analyze particle' function. Total $\beta$ tubulin fluorescence area was directly measured in $\beta$ tubulin fluorescence images, after setting a fixed threshold using the 'analyze particle' function, as described above. Finally, total Hylite-488-A $\beta \quad 1-42 / \beta$ tubulincolocalizing area was normalized to total $\beta$ tubulin area in each field. $\beta$ tubulin was revealed by Alexa-633 fluorophore to avoid significant interference of Hylite488 in the red channel. Quantification of binding was normalized to $\beta$ tubulin towing to the decrease in MAP-2 immunofluorescence staining upon A $\beta$ 1-42 binding.

Maximum projection of confocal stacks in the $x-y$ plane and $z$ axis scans were generated using Image J 1.46r software.

Confocal imaging and measurements of fluorescent fibrils. Hylite-488-A $\beta$ 1-42 fibrils (Anaspec) were prepared as described above, overnight exposed or not to MVs and incubated for $1 \mathrm{~h}$ with primary cultures of hippocampal neurons. Neurons were then fixed and stained for MAP-2. Fluorescence images of A $\beta$ 1-42 fibrils were acquired with a Leica SPE confocal microscope by an operator blinded to the study and analyzed using Image $\mathrm{J} 1.46 \mathrm{r}$ software. A fixed threshold was set on Hylite-488-A $\beta$ 1-42-positive images. After selecting the area parameter $(\mu \mathrm{m})$ at 0.1 -infinite, the area of single fibrils was automatically measured using the 'analyze particle' function. The percentage of fibrils with increasing area values - at intervals of $5 \mu \mathrm{m}^{2}$ - was calculated and the cumulative distribution plot was constructed using OriginPro 8 software (Microcal INC., Claymont, DE, USA).

Western blotting. Lysates of shed MVs and exosomes were separated by electrophoresis, blotted on nitrocellulose membrane and revealed using streptavidin (1:1500, Sigma), rabbit anti-alix (1:1000, Covalab, Billerica, MA, USA) and mouse anti-Tsg101 (1:1000, Abcam, Cambridge, UK). Immunoreactive bands were detected using SuperSignal West Femto Pierce ECL (Thermo Fisher Scientific Inc., Rockford, IL, USA) and ECL film (Amersham, GE Healthcare Limited, Upsala, Sweden).

Endogenous glutamate determination. Endogenous glutamate content was measured by high-performance liquid chromatography analysis following precolumn derivatization with o-phthalaldehyde and discontinuous triphase gradient separation on a C18 reverse-phase chromatographic column $\left(10 \times 4.6 \mathrm{~mm}^{2}\right.$, $3 \mu \mathrm{m}$; at $30^{\circ} \mathrm{C}$; Chrompack, Middleburg, The Netherlands) coupled with fluorometric detection (excitation wavelength $350 \mathrm{~nm}$; emission wavelength $450 \mathrm{~nm}$ ). Homoserine was used as an internal standard. ${ }^{63}$

SELDI-TOF mass spectrometry. The immune-proteomic assay for $A \beta$ isoforms detection was performed as previously reported. ${ }^{64}$ Briefly, $3 \mu \mathrm{l}$ of the specific monoclonal antibodies (6E10 + 4G8) (Covance) at total mAbs concentration of $0.125 \mathrm{mg} / \mathrm{ml}$ (concentration of each $\mathrm{mAb} 0.0625 \mathrm{mg} / \mathrm{ml}$ ) were incubated in a humidity chamber for $2 \mathrm{~h}$ at RT to allow covalent binding to the PS20 ProteinChip Array (Bio-Rad, Hercules, CA, USA). Unreacted sites were blocked with Tris- $\mathrm{HCl}$ $0.5 \mathrm{M}, \mathrm{pH} 8$ in a humid chamber at RT for $30 \mathrm{~min}$. Each spot was first washed three times with PBS containing $0.5 \%(\mathrm{v} / \mathrm{v})$ TritonX-100 and then twice with PBS. The spots were coated with $5 \mu \mathrm{l}$ of sample and incubated in a humid chamber overnight. Each spot was first washed three times with PBS containing $0.1 \%(\mathrm{v} / \mathrm{v})$ 
TritonX-100, twice with PBS and finally with deionized water. One microliter of $\alpha$-cyano4-hydroxy cinnamic acid (Bio-Rad) was added to coated spots. Mass identification was made using the ProteinChip SELDI System, Enterprise Edition (Bio-Rad).

ELISA quantification. Quantitative determination of $A \beta$ 1-42 was performed using innotest ELISA kit (Innogenetics, Gent, Belgium) according to the manufacturer's procedures. Absorbance was detected by 1420 Multilabel Counter Victor 2 (Perkin-Elmer).

Human subjects. Human CSF samples were obtained for diagnostic purposes from subjects with $\mathrm{MCl}(n=53)$, definitive $\mathrm{AD}(n=89)$ according to the Dubois criteria and from age- and sex-matched (Supplementary Table S1) cognitively preserved and neurologically healthy subjects, undergoing spinal anesthesia for orthopedic surgery, serving as controls $(n=20)$. Clinical features of $A D$ and $\mathrm{MCl}$ patients are described in Supplementary Table S1. This research project was approved by the ethical committee of the San Raffaele Scientific Institute, and all subjects signed written informed consent.

Quantification and isolation of MVs from human CSF. Samples of CSF collected by lumbar puncture (200-300 $\mu$ l) were analyzed by flow cytometry, as described previously. ${ }^{30}$ Briefly, human CSF was stained with the myeloid marker IB4-FITC (Sigma). Labeled MVs were quantified within a fixed time interval on a Canto II HTS flow cytometer and analyzed using FCS 3 software. Using sidescatter and forward side-scatter, a vesicle gate was determined over the instrument noise (set by running PBS filtered through a 100-nm filter). Within this gate, IB4-positive events (number of events per ml) were evaluated as a parameter of MV concentration. In a set of experiments, after flow cytometry quantification, human MVs were pelletted at $10000 \times g$ from the volume of CSF yielding $400 \mathrm{MVs}$, which is the amount produced in vitro by $1 \times 10^{6}$ microglia. MVs were then resuspended in neuronal medium and exposed to $1.7 \times 10^{5}$ neurons. Alternatively, MVs $(10000 \times g$ pellet) were processed and analyzed using SELDITOF mass spectrometry.

Statistical analysis. All data are presented as mean \pm S.E. from the indicated number of experiments. Statistical analysis was performed using SigmaStat 3.5 (Jandel Scientific, San Jose, CA, USA) software. After testing data for normal distribution, the appropriate statistical test has been used; see figure legends. The differences are considered to be significant if $P<0.05$ indicated by an asterisk, and those at $P<0.01$ indicated by a double asterisk.

\section{Conflict of Interest}

The authors declare no conflict of interest.

Acknowledgements. We thank Paola Viani (University of Milan) and Elisabetta Menna (CNR Institute of Neuroscience, Milan) for discussion, Annamaria Finardi (San Raffaele Scientific Institute, Milan) for flow cytometry assistance, Maria Rosa Accomazzo (University of Milan) for spectrofluorometer assistance, Maura Francolini and Simona Rodighiero for support with negative staining electron microscopy (Fondazione Filarete, Milan), Marco Milanese (University of Genova) for HPLC measurements, Cinzia Cagnoli and Martina Gabrielli (University of Milan) for help in some experiments. This work was supported by a grant from Fondazione Veronesi to CV, PNR-CNR Aging program 2012-2014, FIRB 2011'RBAP11FRE9_001 to GL and RF, Cariplo 2008-3184 to MM and Ricerca Corrente Italian Ministry of Health to RG.

1. Herring A, Lewejohann L, Panzer AL, Donath A, Kroll O, Sachser N et al. Preventive and therapeutic types of environmental enrichment counteract beta amyloid pathology by different molecular mechanisms. Neurobiol Dis 2011; 42: 530-538.

2. Schilling S, Lauber T, Schaupp M, Manhart S, Scheel E, Bohm G et al. On the seeding and oligomerization of pGlu-amyloid peptides (in vitro). Biochemistry 2006; 45: 12393-12399.

3. Bieschke J, Herbst M, Wiglenda T, Friedrich RP, Boeddrich A, Schiele $F$ et al. Small-molecule conversion of toxic oligomers to nontoxic beta-sheet-rich amyloid fibrils. Nat Chem Biol 2012; 8: 93-101.

4. Winklhofer KF, Tatzelt J, Haass $C$. The two faces of protein misfolding: gain- and loss-offunction in neurodegenerative diseases. EMBO J 2008; 27: 336-349.

5. Benilova I, Karran E, De Strooper B. The toxic Abeta oligomer and Alzheimer's disease: an emperor in need of clothes. Nat Neurosci 2012; 15: 349-357.
6. Lue LF, Brachova L, Civin WH, Rogers J. Inflammation, A beta deposition, and neurofibrillary tangle formation as correlates of Alzheimer's disease neurodegeneration. J Neuropathol Exp Neurol 1996; 55: 1083-1088.

7. Haass C, Mandelkow E. Fyn-tau-amyloid: a toxic triad. Cell 2010; 142: 356-358.

8. Serrano-Pozo A, Mielke ML, Gomez-Isla T, Betensky RA, Growdon JH, Frosch MP et al. Reactive glia not only associates with plaques but also parallels tangles in Alzheimer's disease. Am J Pathol 2011; 179: 1373-1384.

9. Serrano-Pozo A, Mielke ML, Muzitansky A, Gomez-Isla T, Growdon JH, Bacskai BJ et al. Stable size distribution of amyloid plaques over the course of Alzheimer disease. J Neuropathol Exp Neurol 2012; 71: 694-701.

10. Walsh DM, Selkoe DJ. A beta oligomers-a decade of discovery. J Neurochem 2007; 101: 1172-1184.

11. McLean CA, Cherny RA, Fraser FW, Fuller SJ, Smith MJ, Beyreuther K et al. Soluble pool of Abeta amyloid as a determinant of severity of neurodegeneration in Alzheimer's disease. Ann Neurol 1999; 46: 860-866.

12. Mielke MM, Haughey NJ, Bandaru VV, Weinberg DD, Darby E, Zaidi N et al. Plasma sphingomyelins are associated with cognitive progression in Alzheimer's disease. J Alzheimers Dis 2011; 27: 259-269.

13. Martins IC, Kuperstein I, Wilkinson H, Maes E, Vanbrabant M, Jonckheere W et al. Lipids revert inert Abeta amyloid fibrils to neurotoxic protofibrils that affect learning in mice. EMBO J 2008; 27: 224-233.

14. Johansson AS, Garlind A, Berglind-Dehlin F, Karlsson G, Edwards K, Gellerfors P et al. Docosahexaenoic acid stabilizes soluble amyloid-beta protofibrils and sustains amyloidbeta-induced neurotoxicity in vitro. FEBS J 2007; 274: 990-1000.

15. Snyder EM, Nong Y, Almeida CG, Paul S, Moran T, Choi EY et al. Regulation of NMDA receptor trafficking by amyloid-beta. Nat Neurosci 2005; 8: 1051-1058.

16. Lauren J, Gimbel DA, Nygaard HB, Gilbert JW, Strittmatter SM. Cellular prion protein mediates impairment of synaptic plasticity by amyloid-beta oligomers. Nature 2009; 457: $1128-1132$.

17. Um JW, Nygaard HB, Heiss JK, Kostylev MA, Stagi M, Vortmeyer A et al. Alzheimer amyloid-beta oligomer bound to postsynaptic prion protein activates Fyn to impair neurons. Nat Neurosci 2012; 15: 1227-1235.

18. Verdier Y, Zarandi M, Penke B. Amyloid beta-peptide interactions with neuronal and glial cell plasma membrane: binding sites and implications for Alzheimer's disease. J Pept Sci 2004; 10: 229-248.

19. Edison P, Archer HA, Gerhard A, Hinz R, Pavese N, Turkheimer FE et al. Microglia, amyloid, and cognition in Alzheimer's disease: $A n$ [11C](R)PK11195-PET and [11C]PIB-PET study. Neurobiol Dis 2008; 32: 412-419.

20. Okello A, Edison P, Archer HA, Turkheimer FE, Kennedy J, Bullock R et al. Microglial activation and amyloid deposition in mild cognitive impairment: a PET study. Neurology 2009; 72: 56-62.

21. Hollingworth $P$, Harold D, Sims R, Gerrish A, Lambert JC, Carrasquillo MM et al. Common variants at ABCA7, MS4A6A/MS4A4E, EPHA1, CD33 and CD2AP are associated with Alzheimer's disease. Nat Genet 2011; 43: 429-435.

22. Guerreiro R, Wojtas A, Bras J, Carrasquillo M, Rogaeva E, Majounie E et al. TREM2 variants in Alzheimer's disease. N Engl J Med 2013; 368: 117-127.

23. Giulian D, Haverkamp LJ, Yu JH, Karshin W, Tom D, Li J et al. Specific domains of beta-amyloid from Alzheimer plaque elicit neuron killing in human microglia. $J$ Neurosci 1996; 16: 6021-6037.

24. Fuhrmann M, Bittner T, Jung CK, Burgold S, Page RM, Mitteregger G et al. Microglial Cx3cr1 knockout prevents neuron loss in a mouse model of Alzheimer's disease. Nat Neurosci 2010; 13: 411-413.

25. Tan B, Choi RH, Chin TJ, Kaur C, Ling EA. Manipulation of microglial activity as a therapy for Alzheimer's disease. Front Biosci 2012; 4: 1402-1412.

26. Weitz TM, Town T. Microglia in Alzheimer's Disease: it's all about context. Int J Alzheimers Dis 2012; 2012: 314185.

27. Bianco F, Pravettoni E, Colombo A, Schenk U, Moller T, Matteoli M et al. induces vesicle shedding and IL-1 beta release from microglia. J Immunol 2005; 174: 7268-7277.

28. Del Conde I, Shrimpton CN, Thiagarajan P, Lopez JA. Tissue-factor-bearing microvesicles arise from lipid rafts and fuse with activated platelets to initiate coagulation. Blood 2005; 106: 1604-1611.

29. Gonnord P, Delarasse C, Auger R, Benihoud K, Prigent M, Cuif MH et al. Palmitoylation of the P2X7 receptor, an ATP-gated channel, controls its expression and association with lipid rafts. FASEB J 2009; 23: 795-805.

30. Verderio C, Muzio L, Turola E, Bergami A, Novellino L, Ruffini $F$ et al. Myeloid microvesicles are a marker and therapeutic target for neuroinflammation. Ann Neurol 2012; 72: 610-624.

31. Colombo E, Borgiani B, Verderio C, Furlan R. Microvesicles: novel biomarkers for neurological disorders. Front Physiol 2012; 3: 63.

32. Rajendran L, Honsho M, Zahn TR, Keller P, Geiger KD, Verkade P et al. Alzheimer's disease beta-amyloid peptides are released in association with exosomes. Proc Natl Acad Sci USA 2006; 103: 11172-11177.

33. Perry VH, Nicoll JA, Holmes C. Microglia in neurodegenerative disease. Nat Rev Neurol 2010; 6: 193-201.

34. Fukunaga S, Ueno H, Yamaguchi T, Yano Y, Hoshino M, Matsuzaki K. GM1 cluster mediates formation of toxic Abeta fibrils by providing hydrophobic environments. Biochemistry 2012; 51: 8125-8131. 
35. Turola E, Furlan R, Bianco F, Matteoli M, Verderio C. Microglial microvesicle secretion and intercellular signaling. Front Physiol 2012; 3: 149.

36. Jana A, Pahan K. Fibrillar amyloid-beta-activated human astroglia kill primary human neurons via neutral sphingomyelinase: implications for Alzheimer's disease. J Neurosci 2010; 30: 12676-12689.

37. Alberdi E, Sanchez-Gomez MV, Cavaliere F, Perez-Samartin A, Zugaza JL, Trullas R et al. Amyloid beta oligomers induce $\mathrm{Ca} 2+$ dysregulation and neuronal death through activation of ionotropic glutamate receptors. Cell Calcium 2010; 47: 264-272.

38. Bianco F, Perrotta C, Novellino L, Francolini M, Riganti L, Menna E et al. Acid sphingomyelinase activity triggers microparticle release from glial cells. EMBO J 2009; 28 : 1043-1054.

39. Holtzman DM. CSF biomarkers for Alzheimer's disease: current utility and potential future use. Neurobiol Aging 2011; 32(Suppl 1): S4-S9.

40. Yuyama K, Sun H, Mitsutake S, Igarashi Y. Sphingolipid-modulated exosome secretion promotes clearance of amyloid-beta by microglia. J Biol Chem 2012; 287: 10977-10989.

41. Thery C, Ostrowski M, Segura E. Membrane vesicles as conveyors of immune responses. Nat Rev Immunol 2009; 9: 581-593.

42. Mattei V, Barenco MG, Tasciotti V, Garofalo T, Longo A, Boller K et al. Paracrine diffusion of $\operatorname{Pr} P(C)$ and propagation of prion infectivity by plasma membrane-derived microvesicles. PLOS One 2009; 4: e5057.

43. Han X, Fagan AM, Cheng H, Morris JC, Xiong C, Holtzman DM. Cerebrospinal fluid sulfatide is decreased in subjects with incipient dementia. Ann Neurol 2003; 54: 115-119.

44. Malnar M, Kosicek M, Bene R, Tarnik IP, Pavelin S, Babic I et al. Use of cerebrospinal fluid biomarker analysis for improving Alzheimer's disease diagnosis in a non-specialized setting. Acta Neurobiol Exp 2012; 72: 264-271.

45. Ghidoni R, Paterlini A, Albertini V, Glionna M, Monti E, Schiaffonati L et al. Cystatin C is released in association with exosomes: a new tool of neuronal communication which is unbalanced in Alzheimer's disease. Neurobiol Aging 2011; 32: 1435-1442.

46. Vella LJ, Sharples RA, Nisbet RM, Cappai R, Hill AF. The role of exosomes in the processing of proteins associated with neurodegenerative diseases. Eur Biophys $\mathrm{J} 2008$ 37: 323-332.

47. Vingtdeux V, Hamdane M, Begard S, Loyens A, Delacourte A, Beauvillain JC et al. Intracellular $\mathrm{pH}$ regulates amyloid precursor protein intracellular domain accumulation. Neurobiol Dis 2007; 25: 686-696.

48. Yamamoto M, Kiyota T, Walsh SM, Liu J, Kipnis J, Ikezu T. Cytokine-mediated inhibition of fibrillar amyloid-beta peptide degradation by human mononuclear phagocytes. J Immunol 2008; 181: 3877-3886

49. Aguzzi A, Barres BA, Bennett ML. Microglia: scapegoat, saboteur, or something else? Science 2013; 339: 156-161.
50. Prinz M, Priller J, Sisodia SS, Ransohoff RM. Heterogeneity of CNS myeloid cells and their roles in neurodegeneration. Nat Neurosci 2011; 14: 1227-1235.

51. Paresce DM, Chung H, Maxfield FR. Slow degradation of aggregates of the Alzheimer's disease amyloid beta-protein by microglial cells. J Biol Chem 1997; 272: 29390-29397.

52. Lee CY, Landreth GE. The role of microglia in amyloid clearance from the AD brain. J Neural Transm 2010; 117: 949-960.

53. Rajendran L, Annaert W. Membrane trafficking pathways in Alzheimer's disease. Traffic 2012; 13: 759-770.

54. Ariga T, Kobayashi K, Hasegawa A, Kiso M, Ishida H, Miyatake T. Characterization of high-affinity binding between gangliosides and amyloid beta-protein. Arch Biochem Biophys 2001; 388: 225-230.

55. Kiyota T, Yamamoto M, Xiong H, Lambert MP, Klein WL, Gendelman HE et al. CCL2 accelerates microglia-mediated Abeta oligomer formation and progression of neurocognitive dysfunction. PLoS One 2009; 4: e6197.

56. Shen B, Wu N, Yang JM, Gould SJ. Protein targeting to exosomes/microvesicles by plasma membrane anchors. J Biol Chem 2011; 286: 14383-14395.

57. Sharples RA, Vella LJ, Nisbet RM, Naylor R, Perez K, Barnham KJ et al. Inhibition of gamma-secretase causes increased secretion of amyloid precursor protein $\mathrm{C}$-terminal fragments in association with exosomes. FASEB J 2008; 22: 1469-1478.

58. Tamboli IY, Barth E, Christian L, Siepmann M, Kumar S, Singh S et al. Statins promote the degradation of extracellular amyloid \{beta\}-peptide by microglia via stimulation of exosome-associated insulin-degrading enzyme (IDE) secretion. J Biol Chem 2010; 285 : 37405-37414

59. Antonucci F, Turola E, Riganti L, Caleo M, Gabrielli M, Perrotta C et al. Microvesicles released from microglia stimulate synaptic activity via enhanced sphingolipid metabolism. EMBO J 2012; 31: 1231-1240.

60. Klein WL. Abeta toxicity in Alzheimer's disease: globular oligomers (ADDLs) as new vaccine and drug targets. Neurochem Int 2002; 41: 345-352.

61. De Felice FG, Wu D, Lambert MP, Fernandez SJ, Velasco PT, Lacor PN et al. Alzheimer's disease-type neuronal tau hyperphosphorylation induced by A beta oligomers. Neurobiol Aging 2008; 29: 1334-1347.

62. Frassoni C, Inverardi F, Coco S, Ortino B, Grumelli C, Pozzi D et al. Analysis of SNAP-25 immunoreactivity in hippocampal inhibitory neurons during development in culture and in situ. Neuroscience 2005; 131: 813-823.

63. Paluzzi S, Alloisio S, Zappettini S, Milanese M, Raiteri L, Nobile M et al. Adult astroglia is competent for $\mathrm{Na}+/ \mathrm{Ca} 2+$ exchanger-operated exocytotic glutamate release triggered by mild depolarization. J Neurochem 2007; 103: 1196-1207.

64. Albertini V, Bruno A, Paterlini A, Lista S, Benussi L, Cereda C et al. Optimization protocol for amyloid-beta peptides detection in human cerebrospinal fluid using SELDI TOF MS. Proteomics Clin Appl 2010; 4: 352-357.

Supplementary Information accompanies this paper on Cell Death and Differentiation website (http://www.nature.com/cdd) 